Discrete Comput Geom 36:21-69 (2006)

DOI: $10.1007 / \mathrm{s} 00454-005-1211-1$

\title{
A Formulation of the Kepler Conjecture
}

\author{
Thomas C. Hales ${ }^{1}$ and Samuel P. Ferguson ${ }^{2}$ \\ ${ }^{1}$ Department of Mathematics, University of Pittsburgh, \\ Pittsburgh, PA 15217, USA \\ hales@pitt.edu \\ ${ }^{2} 5960$ Millrace Court B-303, \\ Columbia, MD 21045, USA \\ samf2@ comcast.net
}

\begin{abstract}
This paper is the second in a series of six papers devoted to the proof of the Kepler conjecture, which asserts that no packing of congruent balls in three dimensions has density greater than the face-centered cubic packing. The top level structure of the proof is described. A compact topological space is described. Each point of this space can be described as a finite cluster of balls with additional combinatorial markings. A continuous function on this compact space is defined. It is proved that the Kepler conjecture will follow if the value of this function is never greater than a given explicit constant.
\end{abstract}

\section{Introduction}

The following papers give a proof of the Kepler conjecture, which asserts that no packing of congruent balls in three-dimensional Euclidean space has density exceeding that of the face-centered cubic (fcc) packing.

A historical overview of the Kepler conjecture is found in the first paper in this series. Since the history of this problem is treated there, this paper does not go into the details of the extensive literature on this problem. We mention that Hilbert included the Kepler conjecture as part of his eighteenth problem [Hi]. L. Fejes Tóth was the first to formulate a plausible strategy for a proof [Fej8]. He also suggested that computers might play a role in the solution of this problem. The historical account also discusses the development of some of the key concepts of this paper.

An expository account of the proof is contained in [Ha12]. A general reference on sphere packings is [CS2]. A general discussion of the computer algorithms that are used in the proof can be found in [Ha14]. Some speculations on the structure of a second- 
generation proof can be found in [Ha13]. Details of computer calculations can be found on the internet at [Ha16].

The first section of this paper gives the top level structure of the proof of the Kepler conjecture. The next two sections describe the fundamental decompositions of space that are needed in the proof. The first decomposition, which is called the $Q$-system, is a collection of simplices that do not overlap. This decomposition was originally inspired by the Delaunay decomposition of space. The other decomposition, which is called the $V$-cell decomposition, is closely related to the Voronoi decomposition of space. In the following section these two decompositions of space are combined into geometrical objects called decomposition stars. The decomposition star is the fundamental geometrical object in the proof of the Kepler conjecture.

The final section of this paper, which was coauthored with Samuel P. Ferguson, describes a particular nonlinear function on the set of all decomposition stars, called the scoring function. The Kepler conjecture reduces to an optimization problem involving this nonlinear function on the set of all decomposition stars. This is an optimization problem in a finite number of variables. The subsequent papers (Papers III-VI) solve that optimization problem.

The choice of the particular scoring function to use was arrived at jointly with Samuel P. Ferguson. He has contributed to this project in many important ways, including the results in Section 7.

Some history of the proof and this paper is as follows. The original proof, as envisioned in 1994 and accomplished in 1998, was divided into a five-step program. As a result, the original papers were called "Sphere Packings, I," "Sphere Packings, II," and so forth. The first two papers in the series were published in an earlier volume of $D C G$. As it turned out, the fourth step "Sphere Packings, IV" is considerably more difficult than the other steps in the program. It became clear that a single paper would not suffice, and the fourth step of the proof was divided into two parts "Sphere Packings, IV" and "Kepler Conjecture (Sphere Packings, VI)." Samuel Ferguson's thesis "Sphere Packings, V" solved one of the five major steps in the proof. (Although "Sphere Packings, IV" and "Sphere Packings, VI" belonged together, because of the numbering scheme, Ferguson's theses "Sphere Packings, V" was inserted between these two papers.)

The proof that is contained in this volume is a rewritten version of the proof. For historical reasons, the papers in this volume have retained the original titles, but because of extensive revisions over the past several years, the proof is no longer arranged according to the five steps of the 1994 program.

In addition to the $5+1$ papers corresponding to the five steps of the original program, there is the current paper. It has the following origin. In 1996 it became clear that progress on the problem required some adjustments in the main nonlinear optimization problem of "Sphere Packings, I" and "II." As the original 1996 manuscript put it, "There are infinitely many scoring schemes that should lead to a proof of the Kepler conjecture. The problem is to formulate the scheme that makes the Kepler conjecture as accessible as possible" [Ha5]. The original purpose of this paper was to make some useful improvements in the scoring function from "Sphere Packings, I" and "II" and to make the changes in such a way that the main results of those papers would still hold true.

Over the past years, this paper has grown considerably in scope to the point that it is now lays the foundation for all of the papers in the series. In fact, all of the foundational 
material from "Sphere Packings, I" and "II," and the 1998 preprint series has been collected together in this article. The scoring function is no longer the same as the one presented in "Sphere Packings, I" and "II." This paper adapts the relevant material from these earlier papers to the current scoring function. This paper has expanded to the point that it is now possible to understand the entire proof of the Kepler conjecture without reading "I" and "II."

\section{The Top-Level Structure of the Proof}

This section describes the structure of the proof of the Kepler conjecture.

\subsection{Statement of Theorems}

Theorem 3.1 (The Kepler Conjecture). No packing of congruent balls in Euclidean three space has density greater than that of the face-centered cubic packing (see Fig. 3.1).

This density is $\pi / \sqrt{18} \approx 0.74$.

The proof of this result is presented in this paper. Here, we describe the top-level outline of the proof and give references to the sources of the details of the proof.

By a packing, we mean an arrangement of congruent balls that are nonoverlapping in the sense that the interiors of the balls are pairwise disjoint. Consider a packing of congruent balls in Euclidean three space. There is no harm in assuming that all the balls have unit radius. The density of a packing does not decrease when balls are added to the packing. Thus, to answer a question about the greatest possible density we may add nonoverlapping balls until there is no room to add further balls. Such a packing is said to be saturated.

Let $\Lambda$ be the set of centers of the balls in a saturated packing. Our choice of radius for the balls implies that any two points in $\Lambda$ have distance at least 2 from each other. We call the points of $\Lambda$ vertices. Let $B(x, r)$ denote the closed ball in Euclidean three space at center $x$ and radius $r$. Let $\delta(x, r, \Lambda)$ be the finite density, defined as the ratio of the volume of $B(x, r, \Lambda)$ to the volume of $B(x, r)$, where $B(x, r, \Lambda)$ is defined as the intersection with $B(x, r)$ of the union of all balls in the packing. Set $\Lambda(x, r)=\Lambda \cap B(x, r)$.

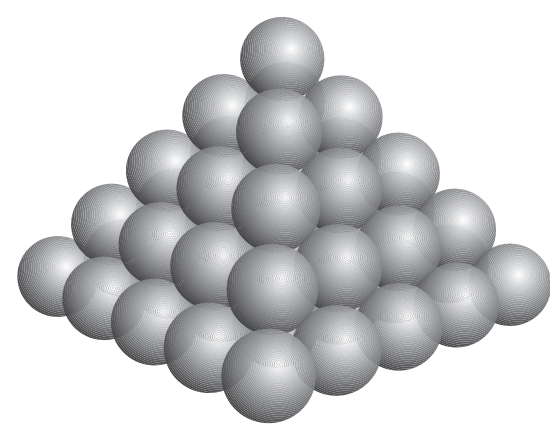

Fig. 3.1. The fcc packing. 
Recall that the Voronoi cell $\Omega(v)=\Omega(v, \Lambda)$ around a vertex $v \in \Lambda$ is the set of points closer to $v$ than to any other ball center. The volume of each Voronoi cell in the fcc packing is $\sqrt{32}$. This is also the volume of each Voronoi cell in the hexagonal-close packing (hcp).

Definition 3.2. Let $A: \Lambda \rightarrow \mathbb{R}$ be a function. We say that $A$ is negligible if there is a constant $C_{1}$ such that for all $r \geq 1$ and all $x \in \mathbb{R}^{3}$,

$$
\sum_{v \in \Lambda(x, r)} A(v) \leq C_{1} r^{2}
$$

We say that the function $A: \Lambda \rightarrow \mathbb{R}$ is fcc-compatible if for all $v \in \Lambda$ we have the inequality

$$
\sqrt{32} \leq \operatorname{vol}(\Omega(v))+A(v) .
$$

The value $\operatorname{vol}(\Omega(v))+A(v)$ may be interpreted as a corrected volume of the Voronoi cell. Fcc-compatibility asserts that the corrected volume of the Voronoi cell is always at least the volume of the Voronoi cells in the fcc and hcp.

Lemma 3.3. If there exists a negligible fcc-compatible function $A: \Lambda \rightarrow \mathbb{R}$ for a saturated packing $\Lambda$, then there exists a constant $C$ such that for all $r \geq 1$ and all $x \in \mathbb{R}^{3}$,

$$
\delta(x, r, \Lambda) \leq \pi / \sqrt{18}+C / r .
$$

The constant $C$ depends on $\Lambda$ only through the constant $C_{1}$.

Proof. The numerator vol $B(x, r, \Lambda)$ of $\delta(x, r, \Lambda)$ is at most the product of the volume of a ball $4 \pi / 3$ with the number $|\Lambda(x, r+1)|$ of balls intersecting $B(x, r)$. Hence

$$
\operatorname{vol} B(x, r, \Lambda) \leq|\Lambda(x, r+1)| 4 \pi / 3 .
$$

In a saturated packing each Voronoi cell is contained in a ball of radius 2 centered at the center of the cell. The volume of the ball $B(x, r+3)$ is at least the combined volume of Voronoi cells whose center lies in the ball $B(x, r+1)$. This observation, combined with fcc-compatibility and negligibility, gives

$$
\begin{aligned}
\sqrt{32}|\Lambda(x, r+1)| & \leq \sum_{v \in \Lambda(x, r+1)}(A(v)+\operatorname{vol}(\Omega(v))) \\
& \leq C_{1}(r+1)^{2}+\operatorname{vol} B(x, r+3) \\
& \leq C_{1}(r+1)^{2}+(1+3 / r)^{3} \operatorname{vol} B(x, r) .
\end{aligned}
$$

Recall that $\delta(x, r, \Lambda)=\operatorname{vol} B(x, r, \Lambda) / \operatorname{vol} B(x, r)$. Divide inequality (3.1) through by $\operatorname{vol} B(x, r)$. Use inequality (3.2) to eliminate $|\Lambda(x, r+1)|$ from the resulting inequality. This gives

$$
\delta(x, r, \Lambda) \leq \frac{\pi}{\sqrt{18}}\left(1+\frac{3}{r}\right)^{3}+C_{1} \frac{(r+1)^{2}}{r^{3} \sqrt{32}} .
$$

The result follows for an appropriately chosen constant $C$. 
An analysis of the preceding proof shows that fcc-compatibility leads to the particular value $\pi / \sqrt{18}$ in the statement of Lemma 3.3. If fcc-compatibility were to be dropped from the hypotheses, any negligible function $A$ would still lead to an upper bound $4 \pi /(3 L)$ on the density of a packing, expressed as a function of a lower bound $L$ on all $\operatorname{vol} \Omega(v)+A(v)$.

Remark 3.4. We take the precise meaning of the Kepler conjecture to be a bound on the essential supremum of the function $\delta(x, r, \Lambda)$ as $r$ tends to infinity. Lemma 3.3 implies that the essential supremum of $\delta(x, r, \Lambda)$ is bounded above by $\pi / \sqrt{18}$, provided a negligible fcc-compatible function can be found. The strategy will be to define a negligible function, and then to solve an optimization problem in finitely many variables to establish that it is fcc-compatible.

Section 6 defines a compact topological space DS (the space of decomposition stars, Definition 6.2) and a continuous function $\sigma$ on that space, which is directly related to packings.

If $\Lambda$ is a saturated packing, then there is a geometric object $D(v, \Lambda)$ constructed around each vertex $v \in \Lambda . D(v, \Lambda)$ depends on $\Lambda$ only through the vertices in $\Lambda$ that are at most a constant distance away from $v$. That constant is independent of $v$ and $\Lambda$. The objects $D(v, \Lambda)$ are called decomposition stars, and the space of all decomposition stars is precisely DS. Section 6.2 shows that the data in a decomposition star are sufficient to determine a Voronoi cell $\Omega(D)$ for each $D \in$ DS. The same section shows that the Voronoi cell attached to $D$ is related to the Voronoi cell of $v$ in the packing by relation

$$
\operatorname{vol} \Omega(v)=\operatorname{vol} \Omega(D(v, \Lambda)) .
$$

Section 7 defines a continuous real-valued function $A_{0}:$ DS $\rightarrow \mathbb{R}$ that assigns a "weight" to each decomposition star. The topological space DS embeds into a finite-dimensional Euclidean space. The reduction from an infinite-dimensional to a finite-dimensional problem is accomplished by the following results.

Theorem 3.5. For each saturated packing $\Lambda$, and each $v \in \Lambda$, there is a decomposition star $D(v, \Lambda) \in \mathrm{DS}$ such that the function $A: \Lambda \rightarrow \mathbb{R}$ defined by

$$
A(v)=A_{0}(D(v, \Lambda))
$$

is negligible for $\Lambda$.

This is proved as Theorem 7.11. The main object of the proof is then to show that the function $A$ is fcc-compatible. This is implied by the inequality (in a finite number of variables)

$$
\sqrt{32} \leq \operatorname{vol} \Omega(D)+A_{0}(D)
$$

for all $D \in$ DS.

In the proof it is convenient to reframe this optimization problem by composing it with a linear function. The resulting continuous function $\sigma: \mathrm{DS} \rightarrow \mathbb{R}$ is called the scoring function, or score. 
Let $\delta_{\text {tet }}$ be the packing density of a regular tetrahedron. That is, let $S$ be a regular tetrahedron of edge length 2 . Let $B$ be the part of $S$ that lies within distance 1 of some vertex. Then $\delta_{\text {tet }}$ is the ratio of the volume of $B$ to the volume of $S$. We have $\delta_{\text {tet }}=\sqrt{8} \arctan (\sqrt{2} / 5)$.

Let $\delta_{\text {oct }}$ be the packing density of a regular octahedron of edge length 2 , again constructed as the ratio of the volume of points within distance 1 of a vertex to the volume of the octahedron.

The density of the fcc packing is a weighted average of these two ratios

$$
\frac{\pi}{\sqrt{18}}=\frac{\delta_{\mathrm{tet}}}{3}+\frac{2 \delta_{\mathrm{oct}}}{3} \text {. }
$$

This determines the exact value of $\delta_{\text {oct }}$ in terms of $\delta_{\text {tet }}$. We have $\delta_{\text {oct }} \approx 0.72$.

In terms of these quantities,

$$
\sigma(D)=-4 \delta_{\text {oct }}\left(\operatorname{vol}(\Omega(D))+A_{0}(D)\right)+\frac{16 \pi}{3} .
$$

Definition 3.6. We define the constant

$$
p t=4 \arctan \left(\frac{\sqrt{2}}{5}\right)-\frac{\pi}{3} .
$$

Its value is approximately $p t \approx 0.05537$. Equivalent expressions for $p t$ are

$$
p t=\sqrt{2} \delta_{\mathrm{tet}}-\frac{\pi}{3}=-2\left(\sqrt{2} \delta_{\mathrm{oct}}-\frac{\pi}{3}\right) .
$$

In terms of the scoring function $\sigma$, the optimization problem in a finite number of variables (inequality (3.3)) takes the following form. The proof of this inequality is a central concern in this paper.

Theorem 3.7 (Finite-Dimensional Reduction). The maximum of $\sigma$ on the topological space DS of all decomposition stars is the constant $8 p t \approx 0.442989$.

Remark 3.8. The Kepler conjecture is an optimization problem in an infinite number of variables (the coordinates of the points of $\Lambda$ ). The maximization of $\sigma$ on DS is an optimization problem in a finite number of variables. Theorem 3.7 may be viewed as a finite-dimensional reduction of the Kepler conjecture.

Let $t_{0}=1.255\left(2 t_{0}=2.51\right)$. This is a parameter that is used for truncation throughout this paper.

Let $U(v, \Lambda)$ be the set of vertices in $\Lambda$ at nonzero distance at most $2 t_{0}$ from $v$. From $v$ and a decomposition star $D(v, \Lambda)$ it is possible to recover $U(v, \Lambda)$, which we write as $U(D)$. We can completely characterize the decomposition stars at which the maximum of $\sigma$ is attained. 
Theorem 3.9. Let $D$ be a decomposition star at which the function $\sigma: \mathrm{DS} \rightarrow \mathbb{R}$ attains its maximum. Then the set $U(D)$ of vectors at distance at most $2 t_{0}$ from the center has cardinality twelve. Up to Euclidean motion, $U(D)$ is one of two arrangements: the kissing arrangement of the twelve balls around a central ball in the fcc packing or the kissing arrangement of twelve balls in the hcp.

There is a complete description of all packings in which every sphere center is surrounded by twelve others in various combinations of these two patterns. All such packings are built from parallel layers of the $A_{2}$ lattice. (The $A_{2}$ lattice formed by equilateral triangles, is the optimal packing in two dimensions.) See the first paper I.

\subsection{Basic Concepts in the Proof}

To prove Theorems 3.1,3.7, and 3.9, we wish to show that there is no counterexample. In particular, we wish to show that there is no decomposition star $D$ with value $\sigma(D)>8 p t$. We reason by contradiction, assuming the existence of such a decomposition star. With this in mind, we call $D$ a contravening decomposition star if

$$
\sigma(D) \geq 8 p t .
$$

In much of what follows we tacitly assume that every decomposition star under discussion is a contravening one. Thus, when we say that no decomposition stars exist with a given property, it should be interpreted as saying that no such contravening decomposition stars exist.

To each contravening decomposition star $D$, we associate a (combinatorial) plane graph $G(D)$. A restrictive list of properties of plane graphs is described in Section 18.3. Any plane graph satisfying these properties is said to be tame. All tame plane graphs have been classified. There are several thousand, up to isomorphism. The list appears in [Ha16]. We refer to this list as the archival list of plane graphs.

A few of the tame plane graphs are of particular interest. Every decomposition star attached to the fcc packing gives the same plane graph (up to isomorphism). Call it $G_{\mathrm{fcc}}$. Likewise, every decomposition star attached to the hcp gives the same plane graph $G_{\mathrm{hcp}}$ (see Fig. 3.2).
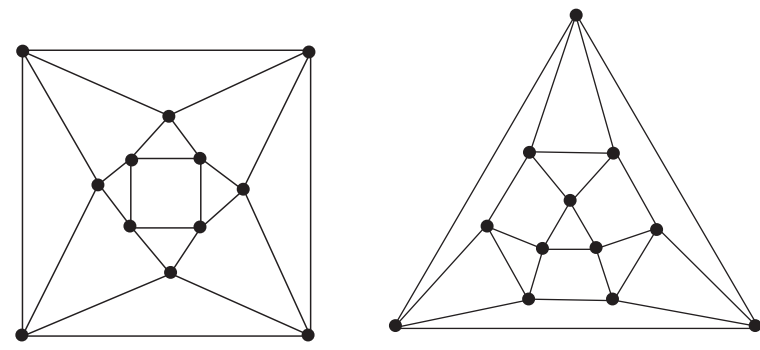

Fig. 3.2. The plane graphs $G_{\mathrm{fcc}}$ and $G_{\mathrm{hcp}}$. 


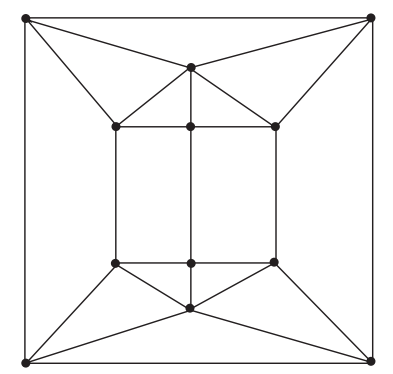

Fig. 3.3. The plane graph $G_{\text {pent }}$ of the pentahedral prism.

There is one more tame plane graph that is particularly troublesome. It is the graph $G_{\text {pent }}$ obtained from the pictured configuration of twelve balls tangent to a given central ball (Fig. 3.3). (Place a ball at the north pole, another at the south pole, and then form two pentagonal rings of five balls.) This case requires individualized attention. S. Ferguson proves the following theorem in Paper V.

Theorem 3.10 (Ferguson). There are no contravening decomposition stars $D$ whose associated plane graph is isomorphic to $G_{\mathrm{pent}}$.

\subsection{Logical Skeleton of the Proof}

Consider the following six claims. Eventually we will give a proof of all six statements. First, we draw out some of their consequences. The main results (Theorems 3.1, 3.7, and 3.9) all follow from these claims.

Claim 3.11. If the maximum of the function $\sigma$ on DS is $8 \mathrm{pt}$, then for every saturated packing $\Lambda$ there exists a negligible fcc-compatible function A.

Claim 3.12. Let $D$ be a contravening decomposition star. Then its plane graph $G(D)$ is tame.

Claim 3.13. If a plane graph is tame, then it is isomorphic to one of the several thousand plane graphs that appear in the archival list of plane graphs.

Claim 3.14. If the plane graph of a contravening decomposition star is isomorphic to one in the archival list of plane graphs, then it is isomorphic to one of the following three plane graphs: $G_{\mathrm{pent}}, G_{\mathrm{hcp}}$, or $G_{\mathrm{fcc}}$.

Claim 3.15. There do not exist any contravening decomposition stars $D$ whose associated graph is isomorphic to $G_{\text {pent }}$. 
Claim 3.16. Contravening decomposition stars exist. If $D$ is a contravening decomposition star, and if the plane graph of $D$ is isomorphic to $G_{\mathrm{fcc}}$ or $G_{\mathrm{hcp}}$, then $\sigma(D)=8 \mathrm{pt}$. Moreover, up to Euclidean motion, $U(D)$ is the kissing arrangement of the twelve balls around a central ball in the fcc packing or the kissing arrangement of twelve balls in the hcp.

Next, we state some of the consequences of these claims.

Lemma 3.17. Assume Claims 3.12-3.15. If $D$ is a contravening decomposition star, then its plane graph $G(D)$ is isomorphic to $G_{\mathrm{hcp}}$ or $G_{\mathrm{fcc}}$.

Proof. Assume that $D$ is a contravening decomposition star. Then its plane graph is tame, and consequently appears on the archival list of plane graphs. Thus, it must be isomorphic to one of $G_{\mathrm{fcc}}, G_{\mathrm{hcp}}$, or $G_{\mathrm{pent}}$. The final graph is ruled out by Claim 3.15.

Lemma 3.18. Assume Claims 3.12-3.16. Then Theorem 3.7 holds.

Proof. By Claim 3.16 and Lemma 3.17, the value $8 p t$ lies in the range of the function $\sigma$ on DS. Assume for a contradiction that there exists a decomposition star $D \in$ DS that has $\sigma(D)>8 p t$. By definition, this is a contravening star. By Lemma 3.17, its plane graph is isomorphic to $G_{\text {hcp }}$ or $G_{\text {fcc }}$. By Claim 3.16, $\sigma(D)=8 p t$, in contradiction with $\sigma(D)>8 p t$.

Lemma 3.19. Assume Claims 3.12-3.16. Then Theorem 3.9 holds.

Proof. By Theorem 3.7, the maximum of $\sigma$ on DS is $8 p t$. Let $D$ be a decomposition star at which the maximum $8 p t$ is attained. Then $D$ is a contravening star. Lemma 3.17 implies that the plane graph is isomorphic to $G_{\mathrm{hcp}}$ or $G_{\mathrm{fcc}}$. The hypotheses of Claim 3.16 are satisfied. The conclusion of Claim 3.16 is the conclusion of Theorem 3.9.

Lemma 3.20. Assume Claims 3.11-3.16. Then the Kepler conjecture (Theorem 3.1) holds.

Proof. As pointed out in Remark 3.4, the precise meaning of the Kepler conjecture is for every saturated packing $\Lambda$, the essential supremum of $\delta(x, r, \Lambda)$ is at most $\pi / \sqrt{18}$.

Let $\Lambda$ be the set of centers of a saturated packing. Let $A: \Lambda \rightarrow \mathbb{R}$ be the negligible, fcc-compatible function provided by Claim 3.11 (and Lemma 3.18). By Lemma 3.3, the function $A$ leads to a constant $C$ such that for all $r \geq 1$ and all $x \in \mathbb{R}^{3}$, the density $\delta(x, r, \Lambda)$ satisfies

$$
\delta(x, r, \Lambda) \leq \pi / \sqrt{18}+C / r .
$$

This implies that the essential supremum of $\delta(x, r, \Lambda)$ is at most $\pi / \sqrt{18}$.

Remark 3.21. One other theorem (Theorem 3.5) was stated without proof in Section 3.1. This result was placed there to motivate the other results. However, it is not an immediate consequence of Claims 3.11-3.16. Its proof appears in Theorem 7.11. 


\subsection{Proofs of the Central Claims}

The previous section showed that the main results in Section 3.1 (Theorems 3.1, 3.7, and 3.9) follow from six claims. This section indicates where each of these claims is proved, and mentions a few facts about the proofs.

Claim 3.11 is proved in Theorem 7.14. Claim 3.12 is proved in Theorem 20.20. Claim 3.13, the classification of tame graphs, is proved in Theorem 19.1. By the classification of such graphs, this reduces the proof of the Kepler conjecture to the analysis of the decomposition stars attached to the finite explicit list of tame plane graphs. We will return to Claim 3.14 in a moment. Claim 3.15 is Ferguson's thesis, cited as Theorem 3.10.

Claim 3.16 is the local optimality of the fcc and hcp. In Section 8, the necessary local analysis is carried out to prove Claim 3.16 as Corollary 8.3.

Now we return to Claim 3.14. This claim is proved as Theorem 23.1. The idea of the proof is the following. Let $D$ be a contravening decomposition star with graph $G(D)$. We assume that the graph $G(D)$ is not isomorphic to $G_{\mathrm{fcc}}, G_{\mathrm{hcp}}$, and $G_{\text {pent }}$ and then prove that $D$ is not contravening. This is a case-by-case argument, based on the explicit archival list of plane graphs.

To eliminate these remaining cases, more-or-less generic arguments can be used. A linear program is attached to each tame graph $G$. The linear program can be viewed as a linear relaxation of the nonlinear optimization problem of maximizing $\sigma$ over all decomposition stars with a given tame graph $G$. Because it is obtained by relaxing the constraints on the nonlinear problem, the maximum of the linear problem is an upper bound on the maximum of the original nonlinear problem. Whenever the linear programming maximum is less than $8 \mathrm{pt}$, it can be concluded that there is no contravening decomposition star with the given tame graph $G$. This linear programming approach eliminates most tame graphs.

When a single linear program fails to give the desired bound, it is broken into a series of linear programming bounds, by branch and bound techniques. For every tame plane graph $G$ other than $G_{\mathrm{hcp}}, G_{\mathrm{fcc}}$, and $G_{\mathrm{pent}}$, we produce a series of linear programs that establish that there is no contravening decomposition star with graph $G$.

The proof is organized in the following way. Sections 4-7 introduce the basic definitions. Section 7 gives a proof of Claim 3.11. Section 8 proves Claim 3.16. Sections 9-14 present the fundamental estimates. Sections 18-19 give a proof of Claim 3.13. Sections 20-22 give a proof of Claim 3.12. Sections 23-25 give a proof of Claim 3.14. Claim 3.15 (Ferguson's thesis) appears in Paper V.

\section{Construction of the $Q$-System}

It is useful to separate the parts of space of relatively high packing density from the parts of space with relatively low packing density. The $Q$-system, which is developed in this section, is a crude way of marking off the parts of space where the density is potentially high. The $Q$-system is a collection of simplices whose vertices are points of the packing $\Lambda$. The $Q$-system is reminiscent of the Delaunay decomposition, in the sense of being a collection of simplices with vertices in $\Lambda$. In fact, the $Q$-system is the remnant of an earlier approach to the Kepler conjecture that was based entirely on the Delaunay decomposition (see [Ha2]). However, the $Q$-system differs from the Delaunay 
decomposition in crucial respects. The most fundamental difference is that the $Q$-system, while consisting of nonoverlapping simplices, does not partition all of space.

This section defines the set of simplices in the $Q$-system and proves that they do not overlap. In order to prove this, we develop a long series of lemmas that study the geometry of intersections of various edges and simplices. At the end of this section we give the proof that the simplices in the $Q$-system do not overlap.

\subsection{Description of the $Q$-system}

Fix a packing of balls of radius 1 . We identify the packing with the set $\Lambda$ of its centers. A packing is thus a subset $\Lambda$ of $\mathbb{R}^{3}$ such that for all $v, w \in \Lambda,|v-w|<2$ implies $v=w$. The centers of the balls are called vertices. The term "vertex" will be reserved for this technical usage. A packing is said to be saturated if for every $x \in \mathbb{R}^{3}$, there is some $v \in \Lambda$ such that $|x-v|<2$. Any packing is a subset of a saturated packing. We assume that $\Lambda$ is saturated. The set $\Lambda$ is countably infinite.

Definition 4.1. We define the truncation parameter to be the constant $t_{0}=1.255$. It is used throughout. Informal arguments that led to this choice of constant are described in Paper I.

Precise constructions that rely on the truncation parameter $t_{0}$ will appear below. We will regularly intersect Voronoi cells with balls of radius $t_{0}$ to obtain lower bounds on their volumes. We will regularly disregard vertices of the packing that lie at distance greater than $2 t_{0}$ from a fixed $v \in \Lambda$ to obtain a finite subset of $\Lambda$ (a finite cluster of balls in the packing) that is easier to analyze than the full packing $\Lambda$.

The truncation parameter is the first of many decimal constants that appear. Each decimal constant is an exact rational value, e.g., $2 t_{0}=251 / 100$. They are not to be regarded as approximations of some other value.

Definition 4.2. A quasi-regular triangle is a set $T \subset \Lambda$ of three vertices such that if $v, w \in T$ then $|w-v| \leq 2 t_{0}$.

Definition 4.3. A simplex is a set of four vertices. A quasi-regular tetrahedron is a simplex $S$ such that if $v, w \in S$ then $|w-v| \leq 2 t_{0}$. A quarter is a simplex whose edge lengths $y_{1}, \ldots, y_{6}$ can be ordered to satisfy $2 t_{0} \leq y_{1} \leq \sqrt{8}, 2 \leq y_{i} \leq 2 t_{0}, i=2, \ldots, 6$. If a quarter satisfies the strict inequalities $2 t_{0}<y_{1}<\sqrt{8}$, then we say that it is a strict quarter. We call the longest edge $\{v, w\}$ of a quarter its diagonal. When the quarter is strict, we also say that its diagonal is strict. When the quarter has a distinguished vertex, the quarter is upright if the distinguished vertex is an endpoint of the diagonal, and flat otherwise.

At times, we identify a simplex with its convex hull. We say, for example, that the circumcenter of a simplex is contained in the simplex to mean that the circumcenter is contained in the convex hull of the four vertices. Similar remarks apply to triangles, quasi-regular tetrahedra, quarters, and so forth. We write $|S|$ for the convex hull of $S$ 
when we wish to be explicit about the distinction between $|S|$ and its set of extreme points.

When we wish to give an order on an edge, triangle, simplex, etc., we present the object as an ordered tuple rather than a set. Thus, we refer to both $\left(v_{1}, \ldots, v_{4}\right)$ and $\left\{v_{1}, \ldots, v_{4}\right\}$ as simplices, depending on the needs of the given context.

Definition 4.4. Two manifolds with boundary overlap if their interiors intersect.

Definition 4.5. A set $O$ of six vertices is called a quartered octahedron if there are four pairwise nonoverlapping strict quarters $S_{1}, \ldots, S_{4}$ all having the same diagonal, such that $O$ is the union of the four sets $S_{i}$ of four vertices. (It follows easily that the strict quarters $S_{i}$ can be given a cyclic order with respect to which each strict quarter $S_{i}$ has a face in common with the next, so that a quartered octahedron is literally a octahedron that has been partitioned into four quarters.)

Remark 4.6. A quartered octahedron may have more than one diagonal of length less than $\sqrt{8}$, so its decomposition into four strict quarters need not be unique. The choice of diagonal has no particular importance. Nevertheless, to make things canonical, we pick the diagonal of length less than $\sqrt{8}$ with an endpoint of smallest possible value with respect to the lexicographical ordering on coordinates; that is, with respect to the ordering $\left(y_{1}, y_{2}, y_{3}\right)<\left(y_{1}^{\prime}, y_{2}^{\prime}, y_{3}^{\prime}\right)$, if $y_{i}=y_{i}^{\prime}$, for $i=1, \ldots, k$, and $y_{k+1}<y_{k+1}^{\prime}$. This selection rule for diagonals is fully translation invariant in the sense that if one octahedron is a translate of another (whether or not they belong to the same saturated packing), then the selected diagonal of one is a translate of the selected diagonal of the other.

Definition 4.7. If $\left\{v_{1}, v_{2}\right\}$ is an edge of length between $2 t_{0}$ and $\sqrt{8}$, we say that a vertex $v\left(\neq v_{1}, v_{2}\right)$ is an anchor of $\left\{v_{1}, v_{2}\right\}$ if its distances to $v_{1}$ and $v_{2}$ are at most $2 t_{0}$.

The two vertices of a quarter that are not on the diagonal are anchors of the diagonal, and the diagonal may have other anchors as well.

Definition 4.8. Let $\mathcal{Q}$ be the set of quasi-regular tetrahedra and strict quarters, enumerated as follows. This set is called the $Q$-system. It is canonically associated with a saturated packing $\Lambda$. (The $Q$ stands for quarters and quasi-regular tetrahedra.)

1. All quasi-regular tetrahedra.

2. Every strict quarter such that none of the quarters along its diagonal overlaps any other quasi-regular tetrahedron or strict quarter.

3. Every strict quarter whose diagonal has four or more anchors, as long as there are not exactly four anchors arranged as a quartered octahedron.

4. The fixed choice of four strict quarters in each quartered octahedron.

5. Every strict quarter $\left\{v_{1}, v_{2}, v_{3}, v_{4}\right\}$ whose diagonal $\left\{v_{1}, v_{3}\right\}$ has exactly three anchors $v_{2}, v_{4}, v_{5}$ provided that the following hold (for some choice of indexing).

(a) $\left\{v_{2}, v_{5}\right\}$ is a strict diagonal with exactly three anchors: $v_{1}, v_{3}, v_{4}$.

(b) $d_{24}+d_{25}>\pi$, where $d_{24}$ is the dihedral angle of the simplex $\left\{v_{1}, v_{3}, v_{2}, v_{4}\right\}$ along the edge $\left\{v_{1}, v_{3}\right\}$ and $d_{25}$ is the dihedral angle of the simplex $\left\{v_{1}, v_{3}, v_{2}, v_{5}\right\}$ along the edge $\left\{v_{1}, v_{3}\right\}$. 
No other quasi-regular tetrahedra or strict quarters are included in the $Q$-system $\mathcal{Q}$.

The following theorem is the main result of this section.

Theorem 4.9. For every saturated packing, there exists a uniquely determined $Q$ system. Distinct simplices in the $Q$-system have disjoint interiors.

While proving the theorem, we give a complete classification of the various ways in which one quasi-regular tetrahedron or strict quarter can overlap another.

Having completed our primary purpose of showing that the simplices in the $Q$-system do not overlap, we state the following small lemma. It is an immediate consequence of the definitions, but is nonetheless useful in the sections that follow.

Lemma 4.10. If one quarter along a diagonal lies in the $Q$-system, then all quarters along the diagonal lie in the $Q$-system.

Proof. This is true by construction. Each of the defining properties of a quarter in the $Q$-system is true for one quarter along a diagonal if and only if it is true of all quarters along the diagonal.

\subsection{Geometric Considerations}

Remark 4.11. The primary definitions and constructions of this paper are translation invariant. That is, if $\lambda \in \mathbb{R}^{3}$ and $\Lambda$ is a saturated packing, then $\lambda+\Lambda$ is a saturated packing. If $A: \Lambda \rightarrow \mathbb{R}$ is a negligible fcc-compatible function for $\Lambda$, then $\lambda+v \mapsto A(v)$ is a negligible fcc-compatible function for $\lambda+\Lambda$. If $\mathcal{Q}$ is the $Q$-system of $\Lambda$, then $\lambda+\mathcal{Q}$ is the $Q$-system of $\lambda+\Lambda$. Because of general translational invariance, when we fix our attention on a particular $v \in \Lambda$, we often assume (without loss of generality) that the coordinate system is fixed in such a way that $v$ lies at the origin.

Our simplices are generally assumed to come labeled with a distinguished vertex, fixed at the origin. (The origin will always be at a vertex of the packing.) We number the edges of each simplex $1, \ldots, 6$, so that edges 1,2 , and 3 meet at the origin, and the edges $i$ and $i+3$ are opposite, for $i=1,2$, 3. (See Fig. 4.1.) $S\left(y_{1}, y_{2}, \ldots, y_{6}\right)$ denotes a simplex whose edges have lengths $y_{i}$, indexed in this way. We refer to the endpoints away from the origin of the first, second, and third edges as the first, second, and third vertices.

Definition 4.12. In general, let $\operatorname{dih}(S)$ be the dihedral angle of a simplex $S$ along its first edge. When we write a simplex in terms of its vertices $\left(w_{1}, w_{2}, w_{3}, w_{4}\right)$, then $\left\{w_{1}, w_{2}\right\}$ is understood to be the first edge.

Definition 4.13. We define the radial projection of a set $X$ to be the radial projection $x \mapsto x /|x|$ of $X \backslash 0$ to the unit sphere centered at the origin. We say the two sets cross if their radial projections to the unit sphere overlap. 


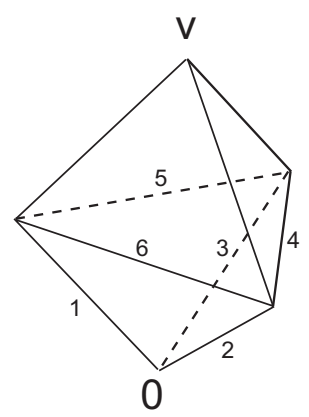

Fig. 4.1. $\mathcal{E}$ measures the distance between the vertices at 0 and $v$. The standard indexing of the edges of a simplex is marked on the lower simplex.

Definition 4.14. If $S$ and $S^{\prime}$ are nonoverlapping simplices with a shared face $F$, we define $\mathcal{E}\left(S, S^{\prime}\right)$ as the distance between the two vertices (one on $S$ and the other on $S^{\prime}$ ) that do not lie on $F$. We may express this as a function

$$
\mathcal{E}\left(S, S^{\prime}\right)=\mathcal{E}\left(S\left(y_{1}, \ldots, y_{6}\right), y_{1}^{\prime}, y_{2}^{\prime}, y_{3}^{\prime}\right)
$$

of nine variables, where $S=S\left(y_{1}, \ldots, y_{6}\right)$ and $S^{\prime}=S\left(y_{1}^{\prime}, y_{2}^{\prime}, y_{3}^{\prime}, y_{4}, y_{5}, y_{6}\right)$, positioned so that $S$ and $S^{\prime}$ are nonoverlapping simplices with a shared face $F$ of edge-lengths $\left(y_{4}, y_{5}, y_{6}\right)$. The function of nine variables is defined only for values $\left(y_{i}, y_{i}^{\prime}\right)$ for which the simplices $S$ and $S^{\prime}$ exist (Fig. 4.1).

Several lemmas in this paper rely on calculations of lower bounds to the function $\mathcal{E}$ in the special case when the edge between the vertices 0 and $v$ passes through the shared face $F$. If intervals containing $y_{1}, \ldots, y_{6}, y_{1}^{\prime}, y_{2}^{\prime}, y_{3}^{\prime}$ are given, then lower bounds on $\mathcal{E}$ over that domain are generally easy to obtain. Detailed examples of calculations of the lower bound of this function can be found in Section 4 of [Ha6].

To work one example, we suppose we are asked to give a lower bound on $\mathcal{E}$ when the simplex $S=S\left(y_{1}, \ldots, y_{6}\right)$ satisfies $y_{i} \geq 2$ and $y_{4}, y_{5}, y_{6} \leq 2 t_{0}$ and $S^{\prime}=S\left(y_{1}^{\prime}, y_{2}^{\prime}, y_{3}^{\prime}, y_{4}\right.$, $\left.y_{5}, y_{6}\right)$ satisfies $y_{i}^{\prime} \geq 2$, for $i=1, \ldots, 3$. Assume that the edge $\{0, v\}$ passes through the face shared between $S$ and $S^{\prime}$, and that $|v|<\sqrt{8}$, where $v$ is the vertex of $S^{\prime}$ that is not on $S$. We claim that any pair $S, S^{\prime}$ can be deformed by moving one vertex at a time until $S$ is deformed into $S\left(2,2,2,2 t_{0}, 2 t_{0}, 2 t_{0}\right)$ and $S^{\prime}$ is deformed into $S\left(2,2,2,2 t_{0}, 2 t_{0}, 2 t_{0}\right)$. Moreover, these deformations preserve the constraints (including that $\{0, v\}$ passes through the shared face), and are nonincreasing in $|v|$. From the existence of this deformation, it follows that the original $|v|$ satisfies

$$
|v| \geq \mathcal{E}\left(S\left(2,2,2,2 t_{0}, 2 t_{0}, 2 t_{0}\right), 2,2,2\right) .
$$

We produce the deformation in this case as follows. We define the pivot of a vertex $v$ with respect to two other vertices $\left\{v_{1}, v_{2}\right\}$ as the circular motion of $v$ held at a fixed distance from $v_{1}$ and $v_{2}$, leaving all other vertices fixed. The axis of the pivot is the line through the two fixed vertices. Each pivot of a vertex can move in two directions. Let 
the vertices of $S$ be $\left\{0, v_{1}, v_{2}, v_{3}\right\}$, labeled so that $\left|v_{i}\right|=y_{i}$. Let $S^{\prime}=\left\{v, v_{1}, v_{2}, v_{3}\right\}$. We pivot $v_{1}$ around the axis through 0 and $v_{2}$. By choice of a suitable direction for the pivot, $v_{1}$ moves away from $v$ and $v_{3}$. Its distance to 0 and $v_{2}$ remains fixed. We continue with this circular motion until $\left|v_{1}-v_{3}\right|$ achieves its upper bound or the segment $\left\{v_{1}, v_{3}\right\}$ intersects the segment $\{0, v\}$ (which threatens the constraint that the segment $\{0, v\}$ must pass through the common face). (We leave it as an exercise ${ }^{2}$ to check that the second possibility cannot occur because of the edge length upper bounds on both diagonals of $\sqrt{8}$. That is, there does not exist a convex planar quadrilateral with sides at least 2 and diagonals less than $\sqrt{8}$.) Thus, $\left|v_{1}-v_{3}\right|$ attains its constrained upper bound $2 t_{0}$. Similar pivots to $v_{2}$ and $v_{3}$ increase the lengths $\left|v_{1}-v_{2}\right|,\left|v_{2}-v_{3}\right|$, and $\left|v_{3}-v_{1}\right|$ to $2 t_{0}$. Similarly, $v$ may be pivoted around the axis through $v_{1}$ and $v_{2}$ to decrease the distance to $v_{3}$ and 0 until the lower bound of 2 on $\left|v-v_{3}\right|$ is attained. Further pivots reduce all remaining edge lengths to 2 . In this way we obtain a rigid figure realizing the absolute lower bound of $|v|$. A calculation with explicit coordinates gives $|v|>2.75$.

Because lower bounds are generally easily determined from a series of pivots through arguments such as this one, we state them without proof. We state that these bounds were obtained by geometric considerations, to indicate that the bounds were obtained by the deformation arguments of this paragraph.

\subsection{Incidence Relations}

Lemma 4.15. Let $v, v_{1}, v_{2}, v_{3}$, and $v_{4}$ be distinct points in $\mathbb{R}^{3}$ with pairwise distances at least 2 . Suppose that $\left|v_{i}-v_{j}\right| \leq 2 t_{0}$ for $i \neq j$ and $\{i, j\} \neq\{1,4\}$. Then $v$ does not lie in the convex hull of $\left\{v_{1}, v_{2}, v_{3}, v_{4}\right\}$.

Proof. This lemma is proved in Lemma 3.5 of [Ha6].

Lemma 4.16. Let $S$ be a simplex whose edges have length between 2 and $2 \sqrt{2}$. Suppose that $v$ has distance at least 2 from each of the vertices of $S$. Then $v$ does not lie in the convex hull of $S$.

Proof. Assume for a contradiction that $v$ lies in the convex hull of $S$. Place a unit sphere around $v$. The simplex $S$ partitions the unit sphere into four spherical triangles, where each triangle is the intersection of the unit sphere with the cone over a face of $S$, centered at $v$. By the constraints on the lengths of edges, the arclength of each edge of the spherical triangle is at most $\pi / 2$. ( $\pi / 2$ is attained when $v$ has distance 2 to two of the vertices, and these two vertices have distance $2 \sqrt{2}$ between them.) A spherical triangle with edges of arclength at most $\pi / 2$ has area at most $\pi / 2$. In fact, any such spherical triangle can be placed inside an octant of the unit sphere, and each octant has area $\pi / 2$. This partitions the sphere of area $4 \pi$ into four regions of area at most $\pi / 2$. This is absurd.

Corollary 4.17. No vertex of the packing is contained in the convex hull of a quasiregular tetrahedron or quarter (other than the vertices of the simplex).

\footnotetext{
${ }^{2}$ Compare Lemma 4.21.
} 
Proof. The corollary is immediate.

Definition 4.18. Let $v_{1}, v_{2}, w_{1}, w_{2}, w_{3} \in \Lambda$ be distinct. We say that an edge $\left\{v_{1}, v_{2}\right\}$ passes through the triangle $\left\{w_{1}, w_{2}, w_{3}\right\}$ if the convex hull of $\left\{v_{1}, v_{2}\right\}$ meets some point of the convex hull of $\left\{w_{1}, w_{2}, w_{3}\right\}$ and if that point of intersection is not any of the extreme points $v_{1}, v_{2}, w_{1}, w_{2}, w_{3}$.

Lemma 4.19. An edge of length $2 t_{0}$ or less cannot pass through a triangle whose edges have lengths $2 t_{0}, 2 t_{0}$, and $\sqrt{8}$ or less.

Proof. The distance between each pair of vertices is at least 2. Geometric considerations show that the edge has length at least

$$
\mathcal{E}\left(S\left(2,2,2,2 t_{0}, 2 t_{0}, \sqrt{8}\right), 2,2,2\right)>2 t_{0}
$$

Definition 4.20. Let $\eta(x, y, z)$ denote the circumradius of a triangle with edge-lengths $x, y$, and $z$.

Lemma 4.21. Suppose that the circumradius of $\left\{v_{1}, v_{2}, v_{3}\right\}$ is less than $\sqrt{2}$. Then an edge $\left\{w_{1}, w_{2}\right\} \subset \Lambda$ of length at most $\sqrt{8}$ cannot pass through the face.

Proof. Assume for a contradiction that $\left\{w_{1}, w_{2}\right\}$ passes through the triangle $\left\{v_{1}, v_{2}, v_{3}\right\}$. By geometric considerations, the minimal length for $\left\{w_{1}, w_{2}\right\}$ occurs when $\left|w_{i}-v_{j}\right|=2$, for $i=1,2, j=1,2,3$. This distance constraint places the circumscribing circle of $\left\{v_{1}, v_{2}, v_{3}\right\}$ on the sphere of radius 2 centered at $w_{1}$ (resp. $w_{2}$ ). If $r<\sqrt{2}$ is the circumradius of $\left\{v_{1}, v_{2}, v_{3}\right\}$, then for this extremal configuration we have the contradiction

$$
\sqrt{8} \geq\left|w_{1}-w_{2}\right|=2 \sqrt{4-r^{2}}>\sqrt{8}
$$

Lemma 4.22. If an edge of length at most $\sqrt{8}$ passes through a quasi-regular triangle, then each of the two endpoints of the edge is at most 2.2 away from each of the vertices of the triangle (see Fig. 4.2).

Proof. Let the diagonal edge be $\left\{v_{0}, v_{0}^{\prime}\right\}$ and let the vertices of the face be $\left\{v_{1}, v_{2}, v_{3}\right\}$. If $\left|v_{i}-v_{0}\right|>2.2$ or $\left|v_{i}-v_{0}^{\prime}\right|>2.2$ for some $i>0$, then geometric considerations give the contradiction

$$
\left|v_{0}-v_{0}^{\prime}\right| \geq \mathcal{E}\left(S\left(2,2,2,2 t_{0}, 2 t_{0}, 2 t_{0}\right), 2,2,2.2\right)>\sqrt{8}
$$

Lemma 4.23. Suppose $S$ and $S^{\prime}$ are quasi-regulartetrahedra that share a face. Suppose that the edge e between the two vertices that are not shared has length at most $\sqrt{8}$. Then the convex hull of $S$ and $S^{\prime}$ consists of three quarters with diagonal e. No other quarter overlaps $S$ or $S^{\prime}$. 

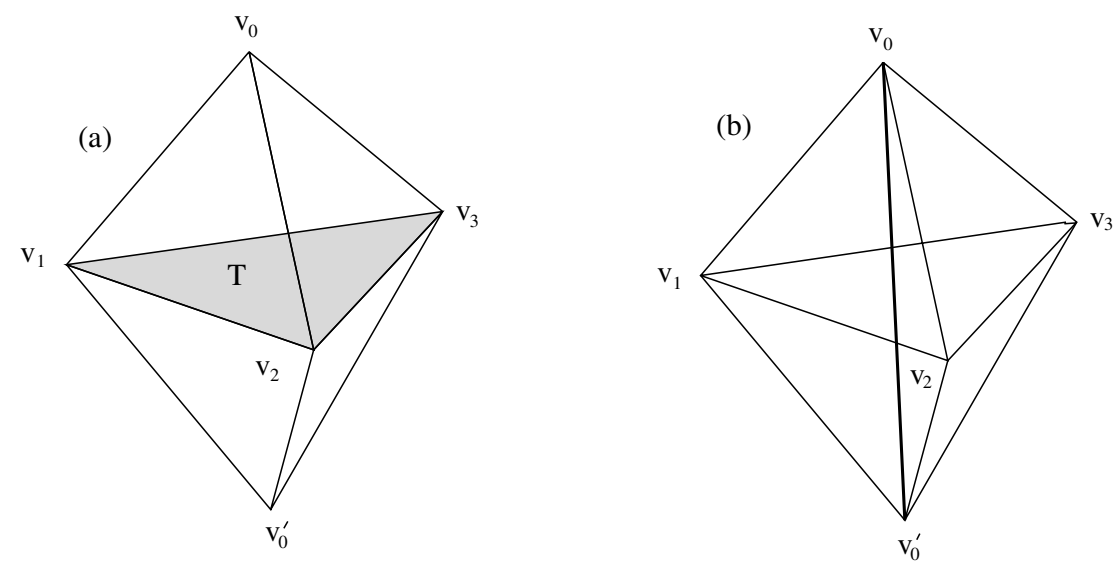

Fig. 4.2. (a) Two quasi-regular tetrahedra that share a face. (b) The same convex body viewed as three quarters that share a diagonal.

Proof. Suppose that $S$ and $S^{\prime}$ are adjacent quasi-regular tetrahedra with a common face $F$. By Lemma 4.22, each of the six external faces of this pair of quasi-regular tetrahedra has circumradius at most $\eta\left(2.2,2.2,2 t_{0}\right)<\sqrt{2}$. A diagonal of a quarter cannot pass through a face of this size by Lemma 4.21. This implies that no other quarter overlaps these quasi-regular tetrahedra.

Lemma 4.24. Suppose an edge $\left\{w_{1}, w_{2}\right\}$ of length at most $\sqrt{8}$ passes through the face formed by a diagonal $\left\{v_{1}, v_{2}\right\}$ and one of its anchors. Then $w_{1}$ and $w_{2}$ are also anchors of $\left\{v_{1}, v_{2}\right\}$.

Proof. This follows from the inequality

$$
\mathcal{E}\left(S\left(2,2,2, \sqrt{8}, 2 t_{0}, 2 t_{0}\right), 2,2,2 t_{0}\right)>\sqrt{8}
$$

and geometric considerations.

Definition 4.25. Let $\Lambda$ be a saturated packing. Assume that the coordinate system is fixed in such a way that the origin is a vertex of the packing. The height of a vertex is its distance from the origin.

Definition 4.26. We say that a vertex is enclosed over a figure if it lies in the interior of the cone at the origin generated by the figure.

Definition 4.27. An adjacent pair of quarters consists of two quarters sharing a face along a common diagonal. The common vertex that does not lie on the diagonal is called the base point of the adjacent pair. (When one of the quarters comes with a marked distinguished vertex, we do not assume that this marked vertex coincides with the base point of the pair.) The other four vertices are called the corners of the configuration. 
Definition 4.28. If the two corners, $v$ and $w$, that do not lie on the diagonal satisfy $|w-v|<\sqrt{8}$, then the base point and four corners can be considered as an adjacent pair in a second way, where $\{v, w\}$ functions as the diagonal. In this case we say that the original diagonal and the diagonal $\{v, w\}$ are conflicting diagonals.

Definition 4.29. A quarter is said to be isolated if it is not part of an adjacent pair. Two isolated quarters that overlap are said to form an isolated pair.

Lemma 4.30. Suppose that there exist four nonzero vertices $v_{1}, \ldots, v_{4}$ of height at most $2 t_{0}$ (that is, $\left|v_{i}\right| \leq 2 t_{0}$ ) forming a skew quadrilateral. Suppose that the diagonals $\left\{v_{1}, v_{3}\right\}$ and $\left\{v_{2}, v_{4}\right\}$ have lengths between $2 t_{0}$ and $\sqrt{8}$. Suppose the diagonals $\left\{v_{1}, v_{3}\right\}$ and $\left\{v_{2}, v_{4}\right\}$ cross. Then the four vertices are the corners of an adjacent pair of quarters with base point at the origin.

Proof. Set $d_{1}=\left|v_{1}-v_{3}\right|$ and $d_{2}=\left|v_{2}-v_{4}\right|$. By hypothesis, $d_{1}$ and $d_{2}$ are at most $\sqrt{8}$. If $\left|v_{1}-v_{2}\right|>2 t_{0}$, geometric considerations give the contradiction

$$
\max \left(d_{1}, d_{2}\right) \geq \mathcal{E}\left(S\left(2 t_{0}, 2,2,2 t_{0}, \sqrt{8}, 2 t_{0}\right), 2,2,2\right)>\sqrt{8} \geq \max \left(d_{1}, d_{2}\right) .
$$

Thus, $\left\{0, v_{1}, v_{2}\right\}$ is a quasi-regular triangle, as are $\left\{0, v_{2}, v_{3}\right\},\left\{0, v_{3}, v_{4}\right\}$, and $\left\{0, v_{4}, v_{1}\right\}$ by symmetry.

Lemma 4.31. If, under the same hypotheses as Lemma 4.30, there is a vertex $w$ of height at most $\sqrt{8}$ enclosed over the adjacent pair of quarters, then $\left\{0, v_{1}, \ldots, v_{4}, w\right\}$ is a quartered octahedron.

Proof. If the enclosed $w$ lies over say $\left\{0, v_{1}, v_{2}, v_{3}\right\}$, then $\left|w-v_{1}\right|,\left|w-v_{3}\right| \leq 2 t_{0}$ (Lemma 4.24), where $\left\{v_{1}, v_{3}\right\}$ is a diagonal. Similarly, the distance from $w$ to the other two corners is at most $2 t_{0}$.

Lemma 4.32. Let $v_{1}$ and $v_{2}$ be anchors of $\{0, w\}$ with $2 t_{0} \leq|w| \leq \sqrt{8}$. If an edge $\left\{v_{3}, v_{4}\right\}$ passes through both faces, $\left\{0, w, v_{1}\right\}$ and $\left\{0, w, v_{2}\right\}$, then $\left|v_{3}-v_{4}\right|>\sqrt{8}$.

Proof. Suppose the figure exists with $\left|v_{3}-v_{4}\right| \leq \sqrt{8}$. Label vertices so $v_{3}$ lies on the same side of the figure as $v_{1}$. Contract $\left\{v_{3}, v_{4}\right\}$ by moving $v_{3}$ and $v_{4}$ until $\left\{v_{i}, u\right\}$ has length 2, for $u=0, w, v_{i-2}$, and $i=3$, 4. Pivot $w$ away from $v_{3}$ and $v_{4}$ around the axis $\left\{v_{1}, v_{2}\right\}$ until $|w|=\sqrt{8}$. Contract $\left\{v_{3}, v_{4}\right\}$ again. By stretching $\left\{v_{1}, v_{2}\right\}$, we obtain a square of edge two and vertices $\left\{0, v_{3}, w, v_{4}\right\}$. Short calculations based on explicit formulas for the dihedral angle and its partial derivatives give

$$
\begin{aligned}
\operatorname{dih}\left(S\left(\sqrt{8}, 2, y_{3}, 2, y_{5}, 2\right)\right) & >1.075, \quad y_{3}, y_{5} \in\left[2,2 t_{0}\right], \\
\operatorname{dih}\left(S\left(\sqrt{8}, y_{2}, y_{3}, 2, y_{5}, y_{6}\right)\right) & >1, \quad y_{2}, y_{3}, y_{5}, y_{6} \in\left[2,2 t_{0}\right] .
\end{aligned}
$$

Then

$\pi \geq \operatorname{dih}\left(0, w, v_{3}, v_{1}\right)+\operatorname{dih}\left(0, w, v_{1}, v_{2}\right)+\operatorname{dih}\left(0, w, v_{2}, v_{4}\right)>1.075+1+1.075>\pi$.

Therefore, the figure does not exist. 
Lemma 4.33. Two vertices $w, w^{\prime}$ of height at most $\sqrt{8}$ cannot be enclosed over a triangle $\left\{v_{1}, v_{2}, v_{3}\right\}$ satisfying $\left|v_{1}-v_{2}\right| \leq \sqrt{8},\left|v_{1}-v_{3}\right| \leq 2 t_{0}$, and $\left|v_{2}-v_{3}\right| \leq 2 t_{0}$.

Proof. For a contradiction, assume the figure exists. The long edge $\left\{v_{1}, v_{2}\right\}$ must have length at least $2 t_{0}$ by Lemma 4.22. This diagonal has anchors $\left\{0, v_{3}, w, w^{\prime}\right\}$. Assume that the cyclic order of vertices around the line $\left\{v_{1}, v_{2}\right\}$ is $0, v_{3}, w, w^{\prime}$. We see that $\left\{v_{1}, w\right\}$ is too short to pass through $\left\{0, v_{2}, w^{\prime}\right\}$, and $w$ is not inside the simplex $\left\{0, v_{1}, v_{2}, w^{\prime}\right\}$. Thus, the projections of the edges $\left\{v_{2}, w\right\}$ and $\left\{0, w^{\prime}\right\}$ to the unit sphere at $v_{1}$ must intersect. It follows that $\left\{0, w^{\prime}\right\}$ passes through $\left\{v_{1}, v_{2}, w\right\}$, or $\left\{v_{2}, w\right\}$ passes through $\left\{v_{1}, 0, w^{\prime}\right\}$. However, $\left\{v_{2}, w\right\}$ is too short to pass through $\left\{v_{1}, 0, w^{\prime}\right\}$. Thus, $\left\{0, w^{\prime}\right\}$ passes through both $\left\{v_{1}, v_{2}, w\right\}$ and $\left\{v_{1}, v_{2}, v_{3}\right\}$. Lemma 4.32 gives the contradiction $\left|w^{\prime}\right|>\sqrt{8}$.

Lemma 4.34. Let $v_{1}, v_{2}, v_{3}$ be anchors of $\{0, w\}$, where $2 t_{0} \leq|w| \leq \sqrt{8},\left|v_{1}-v_{3}\right| \leq$ $\sqrt{8}$, and the edge $\left\{v_{1}, v_{3}\right\}$ passes through the face $\left\{0, w, v_{2}\right\}$. Then $\min \left(\left|v_{1}-v_{2}\right|, \mid v_{2}-\right.$ $\left.v_{3} \mid\right) \leq 2 t_{0}$. Furthermore, if the minimum is $2 t_{0}$, then $\left|v_{1}-v_{2}\right|=\left|v_{2}-v_{3}\right|=2 t_{0}$.

Proof. Assume min $\geq 2 t_{0}$. As in the proof of Lemma 4.32, we may assume that $\left(0, v_{1}, w, v_{3}\right)$ is a square. We may also assume, without loss of generality, that $\left|w-v_{2}\right|=$ $\left|v_{2}\right|=2 t_{0}$. This forces $\left|v_{2}-v_{i}\right|=2 t_{0}$, for $i=1,3$. This is rigid, and is the unique figure that satisfies the constraints. The lemma follows.

\subsection{Overlap of Simplices}

This section gives a proof of Theorem 4.9 (simplices in the $Q$-system do not overlap). This is accomplished in a series of lemmas. The first of these treats quasi-regular tetrahedra.

Lemma 4.35. A quasi-regular tetrahedron does not overlap any other simplex in the $Q$-system.

Proof. Edges of quasi-regular tetrahedra are too short to pass through the face of another quasi-regular tetrahedron or quarter (Lemma 4.19). If a diagonal of a strict quarter passes through the face of a quasi-regular tetrahedron, then Lemma 4.23 shows that the strict quarter is one of three joined along a common diagonal. This is not one of the enumerated types of strict quarter in the $Q$-system.

Lemma 4.36. A quarter in the Q-system that is part of a quartered octahedron does not overlap any other simplex in the $Q$-system.

Proof. By construction, the quarters that lie along a different diagonal of the octahedron do not belong to the $Q$-system. Edges of length at most $2 t_{0}$ are too short to pass through an external face of the octahedron (Lemma 4.19). A diagonal of a strict quarter cannot pass through an external face either, because of Lemma 4.22. 
Lemma 4.37. Let $Q$ be a strict quarter that is part of an adjacent pair. Assume that $Q$ is not part of a quartered octahedron. If $Q$ belongs to the $Q$-system, then it does not overlap any other simplex in the $Q$-system.

The proof of this lemma will give valuable details about how one strict quarter overlaps another.

Proof. Fix the origin at the base point of an adjacent pair of quarters. We investigate the local geometry when another quarter overlaps one of them. (This happens, for example, when there is a conflicting diagonal in the sense of Definition 4.27.)

Label the base point of the pair of quarters $v_{0}$, and the four corners $v_{1}, v_{2}, v_{3}, v_{4}$, with $\left\{v_{1}, v_{3}\right\}$ the common diagonal. Assume that $\left|v_{1}-v_{3}\right|<\sqrt{8}$.

If two quarters overlap then a face on one of them overlaps a face on the other. By Lemmas 4.33 and 4.32, we actually have that some edge (in fact the diagonal) of each passes through a face of the other. This edge cannot exit through another face by Lemma 4.32 and it cannot end inside the simplex by Corollary 4.17. Thus, it must end at a vertex of the other simplex. We break the proof into cases according to which vertex of the simplex it terminates at. In Case 1 the edge has the base point as an endpoint. In Case 2, the edge has a corner as an endpoint.

Case 1. The edge $\{0, w\}$ passes through the triangle $\left\{v_{1}, v_{2}, v_{3}\right\}$, where $\{0, w\}$ is a diagonal of a strict quarter. Lemma 4.24 implies that $v_{1}$ and $v_{3}$ are anchors of $\{0, w\}$. The only other possible anchors of $\{0, w\}$ are $v_{2}$ or $v_{4}$, for otherwise an edge of length at most $2 t_{0}$ passes through a face formed by $\{0, w\}$ and one of its anchors. If both $v_{2}$ and $v_{4}$ are anchors, then we have a quartered octahedron, which has been excluded by the hypotheses of the lemma. Otherwise, $\{0, w\}$ has at most three anchors: $v_{1}, v_{3}$, and either $v_{2}$ or $v_{4}$. In fact, it must have exactly three anchors, for otherwise there is no quarter along the edge $\{0, w\}$. So there are exactly two quarters along the edge $\{0, w\}$. There are at least four anchors along $\left\{v_{1}, v_{3}\right\}: 0, w, v_{2}$, and $v_{4}$. The quarters along the diagonal $\left\{v_{1}, v_{3}\right\}$ lie in the $Q$-system. (None of these quarters is isolated.) The other two quarters, along the diagonal $\{0, w\}$, are not in the $Q$-system. They form an adjacent pair of quarters (with base point $v_{4}$ or $v_{2}$ ) that has conflicting diagonals, $\{0, w\}$ and $\left\{v_{1}, v_{3}\right\}$, of length at most $\sqrt{8}$.

Case 2. $\left\{v_{2}, v_{4}\right\}$ is a diagonal of length less than $\sqrt{8}$ (conflicting with $\left\{v_{1}, v_{3}\right\}$ ). (Note that if an edge of a quarter passes through the shared face of an adjacent pair of quarters, then that edge must be $\left\{v_{2}, v_{4}\right\}$, so that Cases 1 and 2 are exhaustive.) The two diagonals $\left\{v_{1}, v_{3}\right\}$ and $\left\{v_{2}, v_{4}\right\}$ do not overlap. By symmetry, we may assume that $\left\{v_{2}, v_{4}\right\}$ passes through the face $\left\{0, v_{1}, v_{3}\right\}$. Assume (for a contradiction) that both diagonals have an anchor other than 0 and the corners $v_{i}$. Let the anchor of $\left\{v_{2}, v_{4}\right\}$ be denoted $v_{24}$ and that of $\left\{v_{1}, v_{3}\right\}$ be $v_{13}$. Assume the figure is not a quartered octahedron, so that $v_{13} \neq v_{24}$. By Lemma 4.19, it is impossible to draw the edges $\left\{v_{1}, v_{13}\right\}$ and $\left\{v_{13}, v_{3}\right\}$ between $v_{1}$ and $v_{3}$. In fact, if the edges pass outside the quadrilateral $\left\{0, v_{2}, v_{24}, v_{4}\right\}$, one of the edges of length at most $2 t_{0}$ (that is, $\left\{0, v_{2}\right\},\left\{v_{2}, v_{24}\right\},\left\{v_{24}, v_{4}\right\}$, or $\left\{v_{4}, 0\right\}$ ) violates the lemma applied to the face $\left\{v_{1}, v_{3}, v_{13}\right\}$. If they pass inside the quadrilateral, one of the edges $\left\{v_{1}, v_{13}\right\},\left\{v_{13}, v_{3}\right\}$ violates the lemma applied to the face $\left\{0, v_{2}, v_{4}\right\}$ or $\left\{v_{24}, v_{2}, v_{4}\right\}$. We conclude that at most one of the two diagonals has additional anchors. 
If neither of the two diagonals has more than three anchors, we have nothing more than two overlapping adjacent pairs of quarters along conflicting diagonals. The two quarters along the lower edge $\left\{v_{2}, v_{4}\right\}$ lie in the $Q$-system. Another way of expressing this "lower-edge" condition is to require that the two adjacent quarters $Q_{1}$ and $Q_{2}$ satisfy $\operatorname{dih}\left(Q_{1}\right)+\operatorname{dih}\left(Q_{2}\right)>\pi$, when the dihedral angles are measured along the diagonal. The pair $\left(Q_{1}^{\prime}, Q_{2}^{\prime}\right)$ along the upper edge will have $\operatorname{dih}\left(Q_{1}^{\prime}\right)+\operatorname{dih}\left(Q_{2}^{\prime}\right)<\pi$.

If there is a diagonal with more than three anchors, the quarters along the diagonal with more than three anchors lie in the $Q$-system. Any additional quarters along the diagonal $\left\{v_{2}, v_{4}\right\}$ belong to an adjacent pair. Any additional quarters along the diagonal $\left\{v_{1}, v_{3}\right\}$ cannot intersect the adjacent pair along $\left\{v_{2}, v_{4}\right\}$. Thus, every quarter intersecting an adjacent pair also belongs to an adjacent pair.

In both possibilities of Case 2, the two quarters left out of the $Q$-system correspond to a conflicting diagonal.

Remark 4.38. We have seen in the proof of Lemma 4.37 that if a strict quarter $Q$ overlaps a strict quarter that is part of an adjacent pair, then $Q$ is also part of an adjacent pair. Thus, if an isolated strict quarter overlaps another strict quarter, then both strict quarters are necessarily isolated.

Lemma 4.39. If an isolated strict quarter $Q$ overlaps another strict quarter, then the diagonal of $Q$ has exactly three anchors.

The proof of the lemma will give detailed information about the geometrical configuration that is obtained when an isolated quarter overlaps another strict quarter.

Proof. Assume that there are two strict quarters $Q_{1}$ and $Q_{2}$ that overlap. Following Remark 4.38, assume that neither is adjacent to another quarter. Let $\{0, u\}$ and $\left\{v_{1}, v_{2}\right\}$ be the diagonals of $Q_{1}$ and $Q_{2}$. Suppose the diagonal $\left\{v_{1}, v_{2}\right\}$ passes through a face $\{0, u, w\}$ of $Q_{1}$. By Lemma 4.24, $v_{1}$ and $v_{2}$ are anchors of $\{0, u\}$. Again, either the length of $\left\{v_{1}, w\right\}$ is at most $2 t_{0}$ or the length of $\left\{v_{2}, w\right\}$ is at most $2 t_{0}$, say $\left\{w, v_{2}\right\}$ (by Lemma 4.34). It follows that $Q_{1}=\left\{0, u, w, v_{2}\right\}$ and $\left|v_{1}-w\right| \geq 2 t_{0}$. ( $Q_{1}$ is not adjacent to another quarter.) So $w$ is not an anchor of $\left\{v_{1}, v_{2}\right\}$.

Let $\left\{v_{1}, v_{2}, w^{\prime}\right\}$ be a face of $Q_{2}$ with $w^{\prime} \neq 0, u$. If $\left\{v_{1}, w^{\prime}, v_{2}\right\}$ does not link $\{0, u, w\}$, then $\left\{v_{1}, w^{\prime}\right\}$ or $\left\{v_{2}, w^{\prime}\right\}$ passes through the face $\{0, u, w\}$, which is impossible by Lemma 4.19. So $\left\{v_{1}, v_{2}, w^{\prime}\right\}$ links $\{0, u, w\}$ and an edge of $\{0, u, w\}$ passes through the face $\left\{v_{1}, v_{2}, w^{\prime}\right\}$. It is not the edge $\{u, w\}$ or $\{0, w\}$, for they are too short by Lemma 4.19. So $\{0, u\}$ passes through $\left\{w^{\prime}, v_{1}, v_{2}\right\}$. The only anchors of $\left\{v_{1}, v_{2}\right\}$ (other than $w^{\prime}$ ) are $u$ and 0 (by Lemma 4.32). Either $\left\{u, w^{\prime}\right\}$ or $\left\{w^{\prime}, 0\right\}$ has length at most $2 t_{0}$ by Lemma 4.34, but not both, because this would create a quarter adjacent to $Q_{2}$. By symmetry, $Q_{2}=\left\{v_{1}, v_{2}, w^{\prime}, 0\right\}$ and the length of $\left\{u, w^{\prime}\right\}$ is greater than $2 t_{0}$. By symmetry, $\{0, u\}$ has no other anchors either. This determines the local geometry when there are two quarters that intersect without belonging to an adjacent pair of quarters (see Fig. 4.3). It follows that the two quarters form an isolated pair.

Isolated quarters that overlap another strict quarter do not belong to the $Q$-system. 


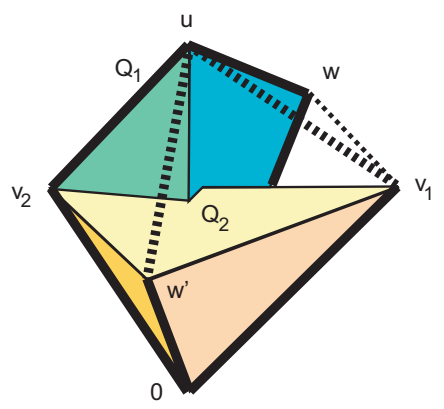

Fig. 4.3. An isolated pair. The isolated pair consists of two simplices $Q_{1}=\left\{0, u, w, v_{2}\right\}$ and $Q_{2}=$ $\left\{0, w^{\prime}, v_{1}, v_{2}\right\}$. The six extremal vertices form an octahedron. This is not a quartered octahedron because the edges $\left\{u, w^{\prime}\right\}$ and $\left\{w, v_{1}\right\}$ have length greater than $2 t_{0}$.

We conclude with the proof of the main theorem of the section.

Proof of Theorem 4.9. The rules defining the $Q$-system specify a uniquely determined set of simplices. The proof that they do not overlap is established by the preceding series of lemmas. Lemma 4.35 shows that quasi-regular tetrahedra do not overlap other simplices in the $Q$-system. Lemma 4.36 shows that the quarters in quartered octahedra are well-behaved. Lemma 4.37 shows that other quarters in adjacent pairs do not overlap other simplices in the $Q$-system. Finally, we treat isolated quarters in Lemma 4.39. These cases cover all possibilities since every simplex in the $Q$-system is a quasi-regular tetrahedron or strict quarter, and every strict quarter is either part of an adjacent pair or isolated.

\section{5. $\quad V$-Cells}

In the proof of the Kepler conjecture we make use of two quite different structures in space. The first structure is the $Q$-system, which was defined in the previous section. It is inspired by the Delaunay decomposition of space and consists of a nonoverlapping collection of simplices that have their vertices at the points of $\Lambda$. Historically, the construction of the nonoverlapping simplices of the $Q$-system grew out of a detailed investigation of the Delaunay decomposition.

The second structure is inspired by the Voronoi decomposition of space. In the Voronoi decomposition, the vertices of $\Lambda$ are the centers of the cells. It is well known that the Voronoi decomposition and Delaunay decomposition are dual to one another. Our modification of Voronoi cells are called $V$-cells.

In general, it is not true that a Delaunay simplex is contained in the union of the Voronoi cells at its four vertices. This incompatibility of structures adds a few complications to Rogers's elegant proof of a sphere packing bound [Ro1]. In this section we show that $V$ cells are compatible with the $Q$-system in the sense that each simplex in the $Q$-system is contained in the union of the $V$-cells at its four vertices (Lemma 5.28). A second compatibility result between these two structures is proved in Lemma 5.29. 
The purpose of this section is to define $V$-cells and to prove the compatibility results just mentioned. In the proof of the Kepler conjecture it is important to keep both structures (the $Q$-system and the $V$-cells) continually at hand. We frequently jump back and forth between these dual descriptions of space in the course of the proof. In Section 6 we define a geometric object (called the decomposition star) around a vertex that encodes both structures. The decomposition star will become our primary object of analysis.

\section{1. $\quad V$-Cells}

Definition 5.1. The Voronoi cell $\Omega(v)$ around a vertex $v \in \Lambda$ is the set of points closer to $v$ than to any other vertex.

Definition 5.2. We construct a set of triangles $\mathcal{B}$ in the packing. The triangles in this set are called barriers. A triangle $\left\{v_{1}, v_{2}, v_{3}\right\}$ with vertices in the packing belongs to $\mathcal{B}$ if and only if one or more of the following properties hold:

1. The triangle is a quasi-regular.

2. The triangle is a face of a simplex in the $Q$-system.

Lemma 5.3. No two barriers overlap; that is, no two open triangular regions of $\mathcal{B}$ intersect.

Proof. If there is overlap, an edge $\left\{w_{1}, w_{2}\right\}$ of one triangle passes through the interior of another $\left\{v_{1}, v_{2}, v_{3}\right\}$. Since $\left|w_{1}-w_{2}\right|<\sqrt{8}$, we have that the circumradius of $\left\{v_{1}, v_{2}, v_{3}\right\}$ is at least $\sqrt{2}$ by Lemma 4.21 and that the length $\left|w_{1}-w_{2}\right|$ is greater than $2 t_{0}$ by Lemma 4.19. If the edge $\left\{w_{1}, w_{2}\right\}$ belongs to a simplex in the $Q$-system, the simplex must be a strict quarter. If $\left\{v_{1}, v_{2}, v_{3}\right\}$ has edge lengths at most $2 t_{0}$, then Lemma 4.22 implies that $\left|w_{i}-v_{j}\right| \leq 2.2$ for $i=1,2$ and $j=1,2,3$. The simplices $\left\{v_{1}, v_{2}, v_{3}, w_{1}\right\}$ and $\left\{v_{1}, v_{2}, v_{3}, w_{2}\right\}$ form a pair of quasi-regular tetrahedra. We conclude that $\left\{v_{1}, v_{2}, v_{3}\right\}$ is a face of a quarter in the $Q$-system. Since, the simplices in the $Q$-system do not overlap, the edge $\left\{w_{1}, w_{2}\right\}$ does not belong to a simplex in the $Q$-system. The result follows.

Definition 5.4. We say that a point $y$ is obstructed at $x \in \mathbb{R}^{3}$ if the line segment from $x$ to $y$ passes through the interior of a triangular region in $\mathcal{B}$. Otherwise, $y$ is unobstructed at $x$. The "obstruction" relation between $x$ and $y$ is clearly symmetric.

For each $w \in \Lambda$, let $I_{w}$ be the cube of side 4, with edges parallel to the coordinate axes, centered at $w$. Thus,

$$
I_{0}=\left\{\left(y_{1}, y_{2}, y_{3}\right):\left|y_{i}\right| \leq 2, i=1,2,3\right\} .
$$

$I_{w}$ has diameter $4 \sqrt{3}$ and $I_{w} \subset B(w, 2 \sqrt{3})$. Let $\mathbb{R}^{3 \prime}$ be the subset of $x \in \mathbb{R}^{3}$ for which $x$ is not equidistant from any two $v, w \in \Lambda(x, 2 \sqrt{3})=B(x, 2 \sqrt{3}) \cap \Lambda$. The subset $\mathbb{R}^{3 \prime}$ is dense in $\mathbb{R}^{3}$, and is obtained locally around a point $x$ by removing finitely many planes (perpendicular bisectors of $\{v, w\}$, for $v, w \in B(x, 2 \sqrt{3})$ ). For $x \in \mathbb{R}^{3 \prime}$, the vertices of $\Lambda(x, 2 \sqrt{3})$ can be strictly ordered by their distance to $x$. 
Definition 5.5. Let $\Lambda$ be a saturated packing. We define a map $\varphi: \mathbb{R}^{3 \prime} \rightarrow \Lambda$ such that the image of $x$ lies in $\Lambda(x, 2 \sqrt{3})$. If $x \in \mathbb{R}^{3 \prime}$, let

$$
\Lambda_{x}=\left\{w \in \Lambda: x \in I_{w} \text { and } w \text { is unobstructed at } x\right\} .
$$

If $\Lambda_{x}=\emptyset$, then let $\varphi(x)$ be the vertex of $\Lambda(x, 2 \sqrt{3})$ closest to $x$. (Since $\Lambda$ is saturated, $\Lambda(x, 2 \sqrt{3})$ is nonempty.) If $\Lambda_{x}$ is nonempty, then let $\varphi(x)$ be the vertex of $\Lambda_{x}$ closest to $x$.

Definition 5.6. For $v \in \Lambda$, let $\operatorname{VC}(v)$ be defined as the closure of $\varphi^{-1}(v)$ in $\mathbb{R}^{3}$. We call it the $V$-cell at $v$.

Remark 5.7. In a saturated packing, the Voronoi cell at $v$ will be contained in a ball centered at $v$ of radius 2. Hence $I_{v}$ contains the Voronoi cell at $v$. By construction, the $V$-cell at $v$ is confined to the cube $I_{v}$. The cubes $I_{v}$ were introduced into the definition of $\varphi$ with the express purpose of forcing $V$-cells to be reasonably small. Had the cubes been omitted from the construction, we would have been drawn to frivolous questions such as whether the closest unobstructed vertex to some $x \in \mathbb{R}^{3}$ might be located in a remote region of the packing.

The set of $V$-cells is our promised decomposition of space.

Lemma 5.8. $\quad V$-cells cover space. The interiors of distinct $V$-cells are disjoint. Each $V$-cell is the closure of its interior.

Proof. The sets $\varphi^{-1}(v)$, for $v \in \Lambda$, cover $\mathbb{R}^{3 \prime}$. Their closures cover $\mathbb{R}^{3}$. The other statements in the lemma follow from the fact that a $V$-cell is a union of finitely many nonoverlapping, closed, convex polyhedra. This is proved in the following lemma.

Lemma 5.9. Each $V$-cell is a finite union of nonoverlapping convex polyhedra.

Proof. During this proof, we ignore sets of measure zero in $\mathbb{R}^{3}$ such as finite unions of planes. Thus, we present the proof as if each point belongs to exactly one Voronoi cell, although this fails on an inconsequential set of measure zero in $\mathbb{R}^{3}$.

It is enough to show that if $E \subset \mathbb{R}^{3}$ is an arbitrary unit cube, then the $V$-cell decomposition of space within $E$ consists of finite unions of nonoverlapping convex polyhedra. Let $X_{E}$ be the set of $w \in \Lambda$ such that $I_{w}$ meets $E$. Included in $X_{E}$ is the set of $w$ whose Voronoi cells cover $E$. The rules for $V$-cells assign $x \in E$ to the $V$-cell centered at some $w \in X_{E}$.

Let $d$ be an upper bound on the distance between a vertex in $X_{E}$ and a point of $E$. By the pythagorean theorem, we may take $d=(1+2) \sqrt{3}$. Let $B_{E}$ be the set of barriers with a vertex at most distance $d$ from some point in $E$.

For each pair $\{u, v\}$ of distinct vertices of $X_{E}$, draw the perpendicular bisecting plane of $\{u, v\}$. Draw the plane through each barrier in $B_{E}$. Draw the plane through 


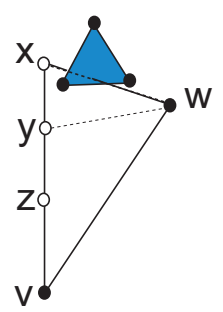

Fig. 5.1. A hypothetical arrangement that leads to a nonconvex $V$-cell at $v$.

each triple $\{u, v, w\}$, where $u \in X_{E}$ and $\{v, w\}$ are two of the vertices of a barrier in $B_{E}$. These finitely many planes partition $E$ into finitely many convex polyhedra. The ranking of distances from $x$ to the points of $X_{E}$ is constant for all $x$ in the interior of any fixed polyhedron. The set of $w \in X_{E}$ that are obstructed at $x$ is constant on the interior of any fixed polyhedron. Thus, by the rules of construction of $V$-cells, for each of these convex polyhedra, there is a $V$-cell that contains it. The result follows.

Remark 5.10. A number of readers of the first version of this manuscript presumed that $V$-cells were necessarily star-convex, in large part because of the inapt name "decomposition star" for a closely related object. The geometry of a $V$-cell is significantly more complex than that of a Voronoi cell. Nowhere do we make a general claim that all $V$-cells are convex, star-convex, or even connected. In Fig. 5.1 we depict a hypothetical case in which the $V$-cell at $v$ is potentially disconnected. (This figure is merely hypothetical, because I have not checked whether it is possible to satisfy all the metric constraints needed for it to exist.) The shaded triangle represents a barrier. The point $x$ is obstructed by the shaded barrier at $w$. If $x$ and $y$ lie closer to $w$ than to $v$, if $v$ is the closest unobstructed vertex to $x$, if $w$ is the closest unobstructed vertex to $y$, if $x, y$, and $z$ are all unobstructed at $v$, and if $z$ lies closer to $v$ than to $w$, then it follows that $x$ and $z$ lie in the $V$-cell at $v$, but that the intervening point $y$ does not. Thus, if all of these conditions are satisfied, the $V$-cell at $v$ is not star-shaped at $v$.

Remark 5.11. Although we have not made a detailed investigation of the subtleties of the geometry of $V$-cells, we face a practical need to give explicit lower bounds on the volume of $V$-cells. Possible geometric pathologies are avoided in the proof by the use of truncation. (To obtain lower bounds on the volume of $V$-cells, parts of the $V$-cell can be discarded.) For example, Lemma 5.23 shows that inside $B\left(v, t_{0}\right)$, the $V$-cell and the Voronoi cell are equal.

In general, truncation will discard points $x$ of $V$-cells where $\Lambda_{x}=\emptyset$. These estimates also discard points of the $V$-cell that are not part of a star-shaped subset of the $V$-cell (to be defined later).

Truncation will be justified later in Lemma 7.18, which shows that the term involving the volume of $V$-cells in the scoring function $\sigma$ has a negative coefficient, so that by decreasing the volume through truncation, we obtain an upper bound on the function $\sigma$. 


\subsection{Orientation}

We introduce the concept of the orientation of a simplex and study its basic properties. The orientation of a simplex will be used to establish various compatibilities between $V$-cells.

Definition 5.12. We say that the orientation of the face of a simplex is negative if the plane through that face separates the circumcenter of the simplex from the vertex of the simplex that does not lie on the face. The orientation is positive if the circumcenter and the vertex lie on the same side of the plane. The orientation is zero if the circumcenter lies in the plane.

Lemma 5.13. At most one face of a quarter $Q$ has negative orientation.

Proof. The proof applies to any simplex with nonobtuse faces. (All faces of a quarter are acute.) Fix an edge and project $Q$ orthogonally to a triangle in a plane perpendicular to that edge. The faces $F_{1}$ and $F_{2}$ of $Q$ along the edge project to edges $e_{1}$ and $e_{2}$ of the triangular projection of $Q$. The line equidistant from the three vertices of $F_{i}$ projects to a line perpendicular to $e_{i}$, for $i=1,2$. These two perpendiculars intersect at the projection of the circumcenter of $Q$. If the faces of $Q$ are nonobtuse, the perpendiculars pass through the segments $e_{1}$ and $e_{2}$ respectively; and the two faces $F_{1}$ and $F_{2}$ cannot both be negatively oriented.

Definition 5.14. Define the polynomial $\chi$ by

$$
\begin{aligned}
\chi\left(x_{1}, \ldots, x_{6}\right)= & x_{1} x_{4} x_{5}+x_{1} x_{6} x_{4}+x_{2} x_{6} x_{5}+x_{2} x_{4} x_{5}+x_{5} x_{3} x_{6} \\
& +x_{3} x_{4} x_{6}-2 x_{5} x_{6} x_{4}-x_{1} x_{4}^{2}-x_{2} x_{5}^{2}-x_{3} x_{6}^{2} .
\end{aligned}
$$

In applications of $\chi$, we have $x_{i}=y_{i}^{2}$, where $\left(y_{1}, \ldots, y_{6}\right)$ are the lengths of the edges of a simplex.

Lemma 5.15. A simplex $S\left(y_{1}, \ldots, y_{6}\right)$ has negative orientation along the face indexed by $(4,5,6)$ if and only if $\chi\left(y_{1}^{2}, \ldots, y_{6}^{2}\right)<0$.

Proof. (This lemma is asserted without proof in [Ha6].) Let $x_{i}=y_{i}^{2}$. Represent the simplex as $S=\left\{0, v_{1}, v_{2}, v_{3}\right\}$, where $\left\{0, v_{i}\right\}$ is the $i$ th edge. Write $n=\left(v_{1}-v_{3}\right) \times\left(v_{2}-\right.$ $v_{3}$ ), a normal to the plane $\left\{v_{1}, v_{2}, v_{3}\right\}$. Let $c$ be the circumcenter of $S$. We can solve for a unique $t \in \mathbb{R}$ such that $c+t n$ lies in the plane $\left\{v_{1}, v_{2}, v_{3}\right\}$. The sign of $t$ gives the orientation of the face $\left\{v_{1}, v_{2}, v_{3}\right\}$. We find by direct calculation that

$$
t=\frac{\chi\left(x_{1}, \ldots, x_{6}\right)}{\sqrt{\Delta\left(x_{1}, \ldots, x_{6}\right)} u\left(x_{4}, x_{5}, x_{6}\right)},
$$

where the terms $\Delta$ and $u$ in the denominator are positive whenever $x_{i}=y_{i}^{2}$, where $\left(y_{1}, \ldots, y_{6}\right)$ are the lengths of edges of a simplex (see Section 8.1 of [Ha6]). Thus, $t$ and $\chi$ have the same sign. The result follows. 
Lemma 5.16. Let $F$ be a set of three vertices. Assume that one edge between pairs of vertices has length between $2 t_{0}$ and $\sqrt{8}$ and that the other two edges have length at most $2 t_{0}$. Let $v$ be any vertex not on $Q$. If the simplex $(F, v)$ has negative orientation along $F$, then it is a quarter.

Proof. The orientation of $F$ is determined by the sign of the function $\chi$ (see Lemma 5.15). The face $F$ is an acute or right triangle. Note that $\partial \chi / \partial x_{1}=x_{4}\left(-x_{4}+x_{5}+x_{6}\right)$. By the law of cosines, $-x_{4}+x_{5}+x_{6} \geq 0$ for an acute triangle. Thus, we have monotonicity in the variable $x_{1}$, and the same is true of $x_{2}$, and $x_{3}$. Also, $\chi$ is quadratic with a negative leading coefficient in each of the variables $x_{4}, x_{5}, x_{6}$. Thus, to check positivity, when any of the lengths is greater than $2 t_{0}$, it is enough to evaluate

$\chi\left(2^{2}, 2^{2}, 4 t_{0}^{2}, x^{2}, y^{2}, z^{2}\right), \quad \chi\left(2^{2}, 4 t_{0}^{2}, 2^{2}, x^{2}, y^{2}, z^{2}\right), \quad \chi\left(4 t_{0}^{2}, 2^{2}, 2^{2}, x^{2}, y^{2}, z^{2}\right)$,

for $x \in\left[2,2 t_{0}\right], y \in\left[2, t_{0}\right]$, and $z \in\left[2 t_{0}, \sqrt{8}\right]$, and verify that these values are nonnegative. (The minimum, which must be attained at a corner of the domain, is 0 .)

Lemma 5.17. Let $\left\{v_{1}, v_{2}, v_{3}\right\}$ be a quasi-regular triangle. Let $v$ be any other vertex. If the simplex $S=\left\{v, v_{1}, v_{2}, v_{3}\right\}$ has negative orientation along $\left\{v_{1}, v_{2}, v_{3}\right\}$, then $S$ is a quasi-regular tetrahedron and $\left|v-v_{i}\right|<2 t_{0}$.

Proof. The proof is similar to the proof of Lemma 5.16. It comes down to checking that

$$
\chi\left(2^{2}, 2^{2}, 4 t_{0}^{2}, x^{2}, y^{2}, z^{2}\right)>0
$$

for $x, y, z \in\left[2,2 t_{0}\right]$.

Lemma 5.18. If a face of a simplex has circumradius less than $\sqrt{2}$, then the orientation is positive along that face.

Proof. If the face has circumradius less than $\sqrt{2}$, by monotonicity

$$
\chi\left(y_{1}^{2}, \ldots, y_{6}^{2}\right) \geq \chi\left(4,4,4, y_{4}^{2}, y_{5}^{2}, y_{6}^{2}\right)=2 y_{4}^{2} y_{5}^{2} y_{6}^{2}\left(2 / \eta\left(y_{4}, y_{5}, y_{6}\right)^{2}-1\right)>0 .
$$

(Here $y_{i}$ are the edge-lengths of the simplex.)

\subsection{Interaction of $V$-cells with the $Q$-system}

We study the structure of one $V$-cell, which we take to be the $V$-cell at the origin $v=0$. Let $\mathcal{Q}$ be the set of simplices in the $Q$-system. For $v \in \Lambda$, let $\mathcal{Q}_{v}$ be the subset of those with a vertex at $v$.

Lemma 5.19. If $x$ lies in the (open) Voronoi cell at the origin, but not in the $V$-cell at the origin, then there exists a simplex $Q \in \mathcal{Q}_{0}$, such that x lies in the cone (at 0 ) over $Q$. Moreover, $x$ does not lie in the interior of $Q$. 
Proof. If $x$ lies in the open Voronoi cell at the origin, then the segment $\{t x: 0 \leq t \leq 1\}$ lies in the Voronoi cell as well. By the definition of the $V$-cell, there is a barrier $\left\{v_{1}, v_{2}, v_{3}\right\}$ that the segment passes through. If the simplex $Q=\left\{0, v_{1}, v_{2}, v_{3}\right\}$ were to have positive orientation with respect to the face $\left\{v_{1}, v_{2}, v_{3}\right\}$, then the circumcenter of $\left\{0, v_{1}, v_{2}, v_{3}\right\}$ would lie on the same side of the plane $\left\{v_{1}, v_{2}, v_{3}\right\}$ as 0 , forcing the intersection of the Voronoi cell with the cone over $Q$ to lie in this same half-space. However, by assumption, $x$ is a point of the Voronoi cell in the opposing half-space. Hence, the simplex $Q$ has negative orientation along $\left\{v_{1}, v_{2}, v_{3}\right\}$.

By construction, the barriers are acute or right triangles. The function $\chi$ (which gives the sign of the orientations of faces) is monotonic in $x_{1}, x_{2}, x_{3}$ when these come from simplices (see the proof of Lemma 5.16.) We consider the implications of negative orientation for each kind of barrier. If the barrier is a quasi-regular triangle, then Lemma 5.17 gives that $Q$ is a quasi-regular tetrahedron when $\chi<0$. If the barrier is a face of a flat quarter in the $Q$-system, then Lemma 5.16 gives that $Q$ is a flat-quarter in the $Q$-system as well. Hence $Q \in \mathcal{Q}_{0}$.

The rest is clear.

Lemma 5.20. If $x$ lies in the open ball of radius $\sqrt{2}$ at the origin, and if $x$ is not in the closed cone over any simplex in $\mathcal{Q}_{0}$, then the origin is unobstructed at $x$.

Proof. Assume for a contradiction that the origin is obstructed by the barrier $T=$ $\{u, v, w\}$ at $x$, and $\{0, u, v, w\}$ is not in $\mathcal{Q}_{0}$. We show that every point in the convex hull of $T$ has distance at least $\sqrt{2}$ from the origin. Since $T$ is a barrier, each edge $\{u, v\}$ has length at most $\sqrt{8}$. Moreover, the heights $|u|$ and $|v|$ are at least 2 , so that every point along each edge of $T$ has distance at least $\sqrt{2}$ from the origin. Suppose that the closest point to the origin in the convex hull of $T$ is an interior point $p$. Reflect the origin through the plane of $T$ to get $w^{\prime}$. The assumptions imply that the edge $\left\{0, w^{\prime}\right\}$ passes through the barrier $T$ and has length less than $\sqrt{8}$. If the barrier $T$ is a quasi-regular triangle, then Lemma 4.22 implies that $\{0, u, v, w\}$ is a quasi-regular tetrahedron in $\mathcal{Q}_{0}$, which is contrary to the hypothesis. Hence $T$ is the face of a quarter in $\mathcal{Q}_{0}$. By Lemma 4.34, one of the simplices $\{0, u, v, w\}$ or $\left\{w^{\prime}, u, v, w\right\}$ is a quarter. Since these are mirror images, both are quarters. Hence $\{0, u, v, w\}$ is a quarter and it is in the $Q$-system by Lemma 4.10. This contradicts the hypothesis of the lemma.

The following corollary is a $V$-cell analogue of a standard fact about Voronoi cells.

Corollary 5.21. The $V$-cell at the origin contains the open unit ball at the origin.

Proof. Let $x$ lie in the open unit ball at the origin. If it is not in the cone over any simplex, then the origin is unobstructed by the lemma, and the origin is the closest point of $\Lambda$. Hence $x \in \operatorname{VC}(0)$. A point in the cone over a simplex $\left\{0, v_{1}, v_{2}, v_{3}\right\} \in \mathcal{Q}_{0}$ lies in $\operatorname{VC}(0)$ if and only if it lies in the set bounded by the perpendicular bisectors of $v_{i}$ and the plane through $\left\{v_{1}, v_{2}, v_{3}\right\}$. The bisectors pose no problem. It is elementary to check that every point of the convex hull of $\left\{v_{1}, v_{2}, v_{3}\right\}$ has distance at least 1 from the origin. (Apply the reflection principle as in the proof of Lemma 5.20 and invoke Lemma 4.19.) 
Lemma 5.22. If $x \in B\left(v, t_{0}\right)$, then $x$ is unobstructed at $v$.

Proof. For a contradiction, supposed that the barrier $T$ obstructs $x$ from the $v$. As in the proof of Lemma 5.20, we find that every edge of $T$ has distance at least $\sqrt{2}$ from the $v$. We may assume that the point of $T$ that is closest to the origin is an interior point. Let $w$ be the reflection of $v$ through $T$. By Lemma 4.19, we have $|v-w|>2 t_{0}$. This implies that every point of $T$ has distance at least $t_{0}$ from $v$. Thus $T$ cannot obstruct $x \in B\left(0, t_{0}\right)$ from $v$.

Lemma 5.23. Inside the ball of radius $t_{0}$ at the origin, the $V$-cell and Voronoi cell coincide:

$$
B\left(0, t_{0}\right) \cap \mathrm{VC}(0)=B\left(0, t_{0}\right) \cap \Omega(0) .
$$

Proof. Let $x \in B\left(0, t_{0}\right) \cap \mathrm{VC}(0) \cap \Omega(v)$, where $v \neq 0$. By Lemma 5.22, the origin is unobstructed at $x$. Thus, $|x-v| \leq|x| \leq t_{0}$. By Lemma 5.22 again, $v$ is unobstructed at $x$, so that $x \in \mathrm{VC}(v)$, contrary to the assumption $x \in \operatorname{VC}(0)$. Thus $B\left(0, t_{0}\right) \cap$ $\mathrm{VC}(0) \subset \Omega(0)$. Similarly, if $x \in B\left(0, t_{0}\right) \cap \Omega(0)$, then $x$ is unobstructed at the origin, and $x \in \mathrm{VC}(0)$.

Definition 5.24. For every pair of vertices $v_{1}, v_{2}$ such that $\left\{0, v_{1}, v_{2}\right\}$ is a quasi-regular triangle, draw a geodesic arc on the unit sphere with endpoints at the radial projections of $v_{1}$ and $v_{2}$. These arcs break the unit sphere into regions called standard regions, as follows. Take the complement of the union of arcs inside the unit sphere. The closure of a connected component of this complement is a standard region. We say that the standard region is triangular if it is bounded by three geodesic arcs, and say that it is nontriangular otherwise.

Lemma 5.25. Let $v_{1}, v_{2}, v_{3}$, and $v_{4}$ be distinct vertices such that $\left|v_{i}\right| \leq 2 t_{0}$ for $i=$ $1,2,3,4$ and $\left|v_{1}-v_{3}\right|,\left|v_{2}-v_{4}\right| \leq 2 t_{0}$. Then the edges $\left\{v_{1}, v_{3}\right\}$ and $\left\{v_{2}, v_{4}\right\}$ do not cross. In particular, the arcs of Definition 5.24 do not meet except at endpoints.

Proof. Exchanging $(1,3)$ with $(2,4)$ if necessary, we may assume for a contradiction that the edge $\left\{v_{1}, v_{3}\right\}$ passes through the face $\left\{0, v_{2}, v_{4}\right\}$. Geometric considerations lead immediately to a contradiction:

$$
2 t_{0}<\mathcal{E}\left(2,2,2,2 t_{0}, 2 t_{0}, 2 t_{0}, 2,2,2\right) \leq\left|v_{1}-v_{3}\right| \leq 2 t_{0} .
$$

Lemma 5.26. Each simplex in the Q-system with a vertex at the origin lies entirely in the closed cone over some standard region $R$.

Proof. Assume for a contradiction that $Q=\left\{0, v_{1}, v_{2}, v_{3}\right\}$ with $v_{1}$ in the open cone over $R_{1}$ and with $v_{2}$ in the open cone over $R_{2}$. Then $\left\{0, v_{1}, v_{2}\right\}$ and $\left\{0, w_{1}, w_{2}\right\}$ (a wall between $R_{1}$ and $R_{2}$ ) overlap; this is contrary to Lemma 5.3. 
Remark 5.27. The next two lemmas help to determine which $V$-cell a given point $x$ belongs to. If $x$ lies in the open cone over a simplex $Q_{0}$ in $\mathcal{Q}$, then Lemma 5.28 describes the $V$-cell decomposition inside $Q$; beyond $Q$ the origin is obstructed by a face of $Q$, so that such an $x$ does not lie in the $V$-cell at 0 . If $x$ does not lie in the open cone over a simplex in $\mathcal{Q}$, but lies in the open cone over a standard region $R$, then Lemma 5.29 describes the $V$-cell. It states, in particular, that, for unobstructed $x$, it can be determined whether $x$ belongs to the $V$-cell at the origin by considering only the vertices $w$ that lie in the closed cone over $R$ (the standard region containing the radial projection of $x$ ). In this sense the intersection of a $V$-cell with the open cone over $R$ is local to the cone over $R$.

Lemma 5.28. If $x$ lies in the interior of a simplex $Q \in \mathcal{Q}$, and if it does not lie on the perpendicular bisector of any edge of $Q$, then it lies in the $V$-cell of the closest vertex of $Q$.

Proof. The segment to any other vertex $v$ crosses a face of the simplex. Such faces are barriers so that $v$ is obstructed at $x$. Thus, the vertices of $Q$ are the only vertices that are not obstructed at $x$.

Let $\mathcal{B}_{0}^{\prime}$ be the set of triangles $T$ such that at least one of the following holds:

- $T$ is a barrier at the origin, or

- $T=\{0, v, w\}$ consists of a diagonal of a quarter in the $Q$-system together with one of its anchors.

Lemma 5.29 (Decoupling Lemma). Let $x \in I_{0}$, the cube of side 4 centered at the origin parallel to coordinate axes. Assume that the closed segment $\{x, w\}$ intersects the closed two-dimensional cone with center 0 over $F=\left\{0, v_{1}, v_{2}\right\}$, where $F \in \mathcal{B}_{0}^{\prime}$. Assume that the origin is not obstructed at $x$. Assume that $x$ is closer to the origin than to both $v_{1}$ and $v_{2}$. Then $x \notin \mathrm{VC}(w)$.

Remark 5.30. The Decoupling Lemma is a crucial result. It permits estimates of the scoring function in Section 7 to be made separately for each standard region. The estimates for separate standard regions are far easier to come by than estimates for the score of the full decomposition star. Eventually, the separate estimates for each standard will be reassembled with linear programming techniques in Section 23.

Proof. (This proof is a minor adaptation of Lemma 2.2 of [Ha7].) Assume for a contradiction that $x$ lies in $\operatorname{VC}(w)$. In particular, we assume that $w$ is not obstructed at $x$. Since the origin is not obstructed at $x, w$ must be closer to $x$ than $x$ is to the origin: $x \cdot w \geq w \cdot w / 2$. The line segment from $x$ to $w$ intersects the closed cone $C(F)$ of the triangle $F=\left\{0, v_{1}, v_{2}\right\}$.

Consider the set $X$ containing $x$ and bounded by the planes $H_{1}$ through $\left\{0, v_{1}, w\right\}$, $H_{2}$ through $\left\{0, v_{2}, w\right\}, H_{3}$ through $\left\{0, v_{1}, v_{2}\right\}, H_{4}=\left\{x: x \cdot v_{1}=v_{1} \cdot v_{1} / 2\right\}$, and $H_{5}=$ $\left\{x: x \cdot v_{2}=v_{2} \cdot v_{2} / 2\right\}$. The planes $H_{4}$ and $H_{5}$ contain the faces of the Voronoi cell at 


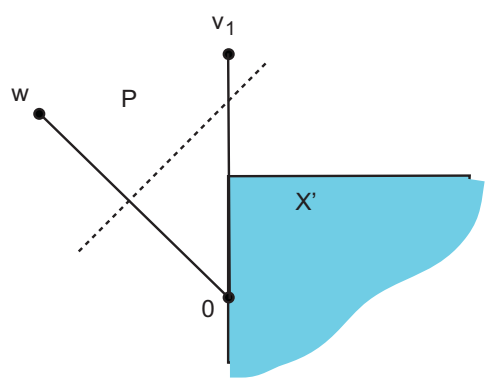

Fig. 5.2. The perpendicular bisector to $\{0, w\}$ (dashed line) cannot meet the quadrant $X^{\prime}$ (shaded).

0 defined by the vertices $v_{1}$ and $v_{2}$. The plane $H_{3}$ contains the triangle $F$. The planes $H_{1}$ and $H_{2}$ bound the set containing points, such as $x$, that can be connected to $w$ by a segment that passes through $C(F)$.

Let $P=\{x: x \cdot w>w \cdot w / 2\}$. The choice of $w$ implies that $X \cap P$ is nonempty. We leave it as an exercise to check that $X \cap P$ is bounded. If the intersection of a bounded polyhedron with a half-space is nonempty, then some vertex of the polyhedron lies in the half-space. Thus, some vertex of $X$ lies in $P$.

We claim that the vertex of $X$ lying in $P$ cannot lie on $H_{1}$. To see this, pick coordinates $\left(x_{1}, x_{2}\right)$ on the plane $H_{1}$ with origin $v_{0}=0$ so that $v_{1}=(0, z)$ (with $\left.z>0\right)$ and $X \cap H_{1} \subset X^{\prime}:=\left\{\left(x_{1}, x_{2}\right): x_{1} \geq 0, x_{2} \leq z / 2\right\}$. See Fig. 5.2. If the quadrant $X^{\prime}$ meets $P$, then the point $v_{1} / 2$ lies in $P$. This is impossible, because every point between 0 and $v_{1}$ lies in the Voronoi cell at 0 or $v_{1}$, and not in the Voronoi cell of $w$. (Recall that for every vertex $v_{1}$ on a barrier at the origin, $\left|v_{1}\right|<\sqrt{8}$.)

Similarly, the vertex of $X$ in $P$ cannot lie on $H_{2}$. Thus, the vertex must be the unique vertex of $X$ that is not on $H_{1}$ or $H_{2}$, namely, the point of intersection of $H_{3}, H_{4}$, and $H_{5}$. This point is the circumcenter $c$ of the face $F$. We conclude that the polyhedron $X_{0}:=X \cap P$ contains $c$. Since $c \in X_{0}$, the simplex $S=\left\{w, v_{1}, v_{2}, 0\right\}$ has nonpositive orientation along the face $\left\{0, v_{1}, v_{2}\right\}$. By Lemmas 5.16 and 5.17, the simplex $S$ lies in $\mathcal{Q}_{0}$.

Let $c$ be the circumcenter of the triangle $F=\left\{0, v_{1}, v_{2}\right\}$ and let $c_{2}$ be the circumcenter of the simplex $\left\{0, v_{1}, v_{2}, w\right\}$. Let $C$ be the convex hull of $\left\{0, v_{1} / 2, v_{2} / 2, c, c_{2}\right\}$. The set $C$ contains the set of points separated from $w$ by the half-plane $H_{3}$, closer to $w$ than to 0 , and closer to 0 than both $v_{1}$ and $v_{2}$. The point $x$ lies in this convex hull $C$. Since this convex hull is nonempty, the simplex $S$ has negative orientation along the face $\left\{0, v_{1}, v_{2}\right\}$.

By assumption, $w$ is not obstructed at $x$. Hence the segment from $w$ to $x$ does not pass through the face $\left\{0, v_{1}, v_{2}\right\}$. The set $C^{\prime}$ of points $y \in C$ such that the segment from $w$ to $y$ does not pass through the face $\left\{0, v_{1}, v_{2}\right\}$ is thus nonempty. The set $C^{\prime}$ must include the extreme point $c_{2}$ of $C$. This means that the plane $\left\{w, v_{1}, v_{2}\right\}$ separates $c_{2}$ from the origin, so that the simplex $S$ has negative orientation also along the face $\left\{w, v_{1}, v_{2}\right\}$. This contradicts Lemma 5.13.

We draw out a simple consequence of the proof. Let $F=\left\{0, v_{1}, v_{2}\right\}$ with edges of length between 2 and $\sqrt{8}$. Let $S=\left\{0, w, v_{1}, v_{2}\right\}$, and assume that $S$ has negative 
orientation along $F$. Let $c$ be the circumcenter of the triangle $F=\left\{0, v_{1}, v_{2}\right\}$ and let $c_{2}$ be the circumcenter of the simplex $\left\{0, v_{1}, v_{2}, w\right\}$. Let $C$ be the convex hull of $\left\{0, v_{1} / 2, v_{2} / 2, c, c_{2}\right\}$. The set $C$ contains the set of points separated from $w$ by the halfplane $H_{3}$, closer to $w$ than to 0 , and closer to 0 than both $v_{1}$ and $v_{2}$. Let $x$ lie in this convex hull $C$.

Lemma 5.31. In this context, $w$ is obstructed at $x$.

Proof. This is what the final paragraph of the previous proof proves by contradiction.

\section{Decomposition Stars}

This section constructs a topological space DS such that each point of DS encodes the geometrical data surrounding a vertex in the packing. The points in this topological space are called decomposition stars. A decomposition star encodes all of the local geometrical information that will be needed in the local analysis of a sphere packing. These geometrical data are sufficiently detailed that it is possible to recover the $V$-cell at $v \in \Lambda$ from the corresponding point in the topological space. It is also possible to recover the simplices in the $Q$-system that have a vertex at $v \in \Lambda$. Thus, a decomposition star has a dual nature that encompasses both the Voronoi-like $V$-cell and the Delaunay-like simplices in the $Q$-system. By encoding both structures, the decomposition star becomes our primary geometric object of analysis.

It can be helpful at times to visualize the decomposition star as a polyhedral object formed by the union of the simplices at $v$ in the $Q$-system with the $V$-cell at $v \in \Lambda$. Although such descriptions can be helpful to the intuition, the formal definition of a decomposition star is rather more combinatorial, expressed as a series of indexing sets that hold the data that are needed to reconstruct the geometry. The formal description of the decomposition star is preferred because it encodes more information than the polyhedral object.

The term "decomposition star" is derived from the earlier term "Delaunay star" that was used in [Ha2] as the name for the union of Delaunay simplices that shared a common vertex. Delaunay stars are star-convex. It is perhaps unfortunate that the term "star" has been retained, because (the geometric realization of) a decomposition star need not be star convex. In fact, Remark 5.10 suggests that $V$-cells can be rather poorly behaved in this respect.

\subsection{Indexing Sets}

We are ready for the formal description of decomposition stars.

Let $\omega=\{0,1,2, \ldots\}$. Pick a bijection $b: \omega \rightarrow \Lambda$ and use this bijection to index the vertices $b(i)=v_{i} \in \Lambda, i=0,1,2 \ldots$ Define the following indexing sets:

- Let $I_{1}=\omega$.

- Let $I_{2}$ be the set of unordered pairs of indices $\{i, j\}$ such that $\left|v_{i}-v_{j}\right| \leq 2 t_{0}=2.51$. 
- Let $I_{3}$ be the set of unordered tuples of indices $\{i, j, k, \ell\}$ such that the corresponding simplex is a strict quarter.

- Let $I_{4}$ be the set of unordered tuples $\{i, j, k, \ell\}$ of indices such that the simplex $\left\{v_{i}, v_{j}, v_{k}, v_{\ell}\right\}$ is in the $Q$-system.

- Let $I_{5}$ be the set of unordered triples $\{i, j, k\}$ of indices such that $v_{i}$ is an anchor of a diagonal $\left\{v_{j}, v_{k}\right\}$ of a strict quarter in the $Q$-system.

- Let $I_{6}$ be the set of unordered pairs $\{i, j\}$ of indices such that the edge $\left\{v_{i}, v_{j}\right\}$ has length in the open interval $\left(2 t_{0}, \sqrt{8}\right)$. (This set includes all such pairs, whether or not they are attached to the diagonal of a strict quarter.)

- Let $I_{7}$ be the set of unordered triples $\{i, j, k\}$ of indices such that the triangle $\left\{v_{i}, v_{j}, v_{k}\right\}$ is a face of a simplex in the $Q$-system and such that the circumradius is less than $\sqrt{2}$.

- Let $I_{8}$ be the set of unordered quadruples $\{i, j, k, \ell\}$ of indices such that the corresponding simplex $\left\{v_{i}, v_{j}, v_{k}, v_{\ell}\right\}$ is a quasi-regular tetrahedron with circumradius less than 1.41.

The data are highly redundant, because some of the indexing sets can be derived from others. However, there is no need to strive for a minimal description of the data.

Set $d_{0}=2 \sqrt{2}+4 \sqrt{3}$. We recall that $\Lambda\left(v, d_{0}\right)=\left\{w \in \Lambda:|w-v| \leq d_{0}\right\}$. Let

$$
T^{\prime}=\left\{i: v_{i} \in \Lambda\left(v, d_{0}\right)\right\} .
$$

It is the indexing set for a neighborhood of $v$.

Fix a vertex $v=v_{a} \in \Lambda$. Let $I_{0}^{\prime}=\{\{a\}\}$. Let

$$
I_{j}^{\prime}=\left\{x \in I_{j}: x \subset T^{\prime}\right\}, \quad \text { for } \quad 1 \leq j \leq 8 .
$$

Each $I_{j}^{\prime}$ is a finite set of finite subsets of $\omega$. Hence $I_{j}^{\prime} \in P(P(\omega))$, where $P(X)$ is the powerset of any set $X$.

Associate with each $v \in \Lambda$ the function $f: T^{\prime} \rightarrow B\left(0, d_{0}\right)$ given by $f(i)=v_{i}-v$, and the tuple

$$
t=\left(I_{0}^{\prime}, \ldots, I_{8}^{\prime}\right) \in P(P(\omega))^{9} .
$$

There is a natural action of the permutation group of $\omega$ on the set of pairs $(f, t)$, where a permutation acts on the domain of $f$ and on $P(P(\omega))$ through its action on $\omega$. Let $[f, t]$ be the orbit of the pair $(f, t)$ under this action. The orbit $[f, t]$ is independent of the bijection $b: \omega \rightarrow \Lambda$. Thus, it is canonically attached to $(v, \Lambda)$.

Definition 6.1. Let $\mathrm{DS}^{\circ}$ be the set of all pairs $[f, t]$ that come from some $v$ in a saturated packing $\Lambda$.

Put a topology on all pairs $(f, t)$ (as we range over all saturated packings $\Lambda$, all choices of indexing $b: \omega \rightarrow \Lambda$, and all $v \in \Lambda$ ) by declaring $(f, t)$ to be close to $\left(f^{\prime}, t^{\prime}\right)$ if and only if $t=t^{\prime}$, domain $(f)=\operatorname{domain}\left(f^{\prime}\right)$, and for all $i \in \operatorname{domain}(f), f(i)$ is close to $f^{\prime}(i)$. That is, we take the topology to be that inherited from the standard topology on $B\left(0, d_{0}\right)$ and the discrete topology on the finite indexing sets. 
The topology on pairs $(f, t)$ descends to the orbit space and gives a topology on $\mathrm{DS}^{\circ}$.

There is a natural compactification of $\mathrm{DS}^{\circ}$ obtained by replacing open conditions by closed conditions. That is, for instance if $\{i, j\}$ is a pair in $I_{6}$, we allow $|f(i)-f(j)|$ to lie in the closed interval $\left[2 t_{0}, \sqrt{8}\right]$. The conditions on each of the other indexing sets $I_{j}$ are similarly relaxed so that they are closed conditions.

Compactness comes from the compactness of the closed ball $B\left(0, d_{0}\right)$, the closed conditions on indexing sets, and the finiteness of $T^{\prime}$.

Definition 6.2. Let DS be the compactification given above of $\mathrm{DS}^{\circ}$. Call it the space of decomposition stars.

Definition 6.3. Let $v$ be a vertex in a saturated packing $\Lambda$. We let $D(v, \Lambda)$ denote the decomposition star attached to $(v, \Lambda)$.

Because of the discrete indexing sets, the space of decomposition stars breaks into a large number of connected components. On each connected component, the combinatorial data are constant. Motion within a fixed connected component corresponds to a motion of a finite set of sphere centers of the packing (in a direction that preserves all of the combinatorial structures).

In a decomposition star, it is no longer possible to distinguish some quasi-regular tetrahedra from quarters solely on the basis of metric relations. For instance, the simplex with edge lengths $2,2,2,2,2,2 t_{0}$ is a quasi-regular tetrahedron and is also in the closure of the set of strict quarters. The indexing set $I_{2}^{\prime}$, which is part of the data of a decomposition star, determines whether the simplex is treated as a quasi-regular tetrahedron or a quarter.

Roughly speaking, two decomposition stars $D(v, \Lambda)$ and $D\left(v^{\prime}, \Lambda^{\prime}\right)$ are close if the translations $\Lambda\left(v, d_{0}\right)-v$ and $\Lambda^{\prime}\left(v^{\prime}, d_{0}\right)-v^{\prime}$ have the same cardinality, and there is a bijection between them that respects all of the indexing sets $I_{j}^{\prime}$ and proximity of vertices.

\subsection{Cells Attached to Decomposition Stars}

To each decomposition star we can associate a $V$-cell centered at 0 by a direct adaptation of Definition 5.6.

Lemma 6.4. The $V$-cell at $v$ depends on $\Lambda$ only through $\Lambda\left(v, d_{0}\right)$ and the indexing sets $I_{j}^{\prime}$.

Proof. We wish to decide whether a given $x$ belongs to the $V$-cell at $v$ or to another contender $w \in \Lambda$. We assume that $x \in I_{v}$, for otherwise $x$ cannot belong to the $V$-cell at $v$. Similarly, we assume $x \in I_{w}$. We must determine whether $v$ or $w$ is obstructed at $x$. For this we must know whether barriers lie on the path between $x$ and $v$ (or $w$ ). Since $|x-w| \leq 2 \sqrt{3}$ and $|x-v| \leq 2 \sqrt{3}$, the point $p$ of intersection of the barrier and the segment $\{x, v\}$ (or $\{x, w\}$ ) satisfies $|x-p| \leq 2 \sqrt{3}$. All the vertices of the barrier then 
have distance at most $\sqrt{8}+2 \sqrt{3}$ from $x$, and hence distance at most $d_{0}=\sqrt{8}+4 \sqrt{3}$ from $v$. The decomposition star $D(v, \Lambda)$ includes all vertices in $\Lambda\left(v, d_{0}\right)$ and the indexing sets of the decomposition star label all the barriers in $\Lambda\left(v, d_{0}\right)$. Thus, the decomposition star at $v$ gives all the data that are needed to determine whether $x \in I_{v}$ belongs to the $V$-cell at $v$.

Corollary 6.5. There is a $V$-cell $\mathrm{VC}(D)$ attached to each decomposition star $D$ such that if $D=D(v, \Lambda)$, then $\operatorname{VC}(D)+v$ is the $V$-cell attached to $(v, \Lambda)$ in Definition 5.6.

Proof. By the lemma the map from $(v, \Lambda)$ maps through the data determining the decomposition star $D(v, \Lambda)$. The definition of $V$-cell extends: the $V$-cell at 0 attached to $[f, t]$ is the set of points in $B\left(0, C_{0}\right)$ for which the origin is the unique closest unobstructed vertex of range $(f)$. The barriers for the obstruction are to be reconstructed from the indexing data sets $I_{j}$ of $t$.

Lemma 6.6. $\operatorname{VC}(D)$ is a finite union of nonoverlapping convex polyhedra. Moreover, $D \mapsto \operatorname{vol}(\operatorname{VC}(D))$ is continuous.

Proof. For the proof, we ignore sets of measure zero, such as finite unions of planes. We may restrict our attention to a single connected component of the space of decomposition stars. On each connected component, the indexing set for each barrier (near the origin) is fixed. The indexing set for the set of vertices near the origin is fixed. For each $D$ the VC-cell breaks into a finite union of convex polyhedra by Lemma 5.9.

As the proof of that lemma shows, some faces of the polyhedra are perpendicular bisecting planes between two vertices near the origin. Such planes vary continuously on (a connected component) of DS. The other faces of polyhedra are formed by planes through three vertices of the packing near the origin. Such planes also vary continuously on DS. It follows that the volume of each convex polyhedron is a continuous function on DS. The sum of these volumes, giving the volume of $\operatorname{VC}(D)$, is also continuous.

Lemma 6.7. Let $\Lambda$ be a saturated packing. The Voronoi cell $\Omega(v)$ at $v$ depends on $\Lambda$ only through $\Lambda\left(v, d_{0}\right)$.

Proof. Let $x$ be an extreme point of the Voronoi cell $\Omega(v)$. The vertex $v$ is one of the vertices closest to $x$. If the distance from $x$ to $v$ is at least 2, then there is room to place another ball centered at $x$ into the packing without overlap. Then $\Lambda$ is not saturated.

Thus, the distance from $x$ to $v$ is less than 2. The Voronoi cell lies in the ball $B(v, 2)$. The Voronoi cell is bounded by the perpendicular bisectors of segments $\{v, w\}$ for $w \in \Lambda$. If $w$ has distance 4 or more from $v$, then the bisector cannot meet the ball $B(v, 2)$ and cannot bound the cell. Since $4<d_{0}$, the proof is complete.

Corollary 6.8. The vertex $v$ and the decomposition star $D(v, \Lambda)$ determine the Voronoi cell at $v$. Infact, the Voronoi cell is determined by $v$ and the first indexing set $I_{1}^{\prime}$ of $D(v, \Lambda)$. 
Definition 6.9. The Voronoi cell $\Omega(D)$ of $D \in$ DS is the set containing the origin bounded by the perpendicular bisectors of $\left\{0, v_{i}\right\}$ for $i \in I_{1}^{\prime}$.

Remark 6.10. It follows from Corollary 6.8 that

$$
\Omega(D(v, \Lambda))=v+\Omega(v) .
$$

In particular, they have the same volume.

Remark 6.11. From a decomposition star $D$, we can recover the set of vertices $U(D)$ of distance at most $2 t_{0}$ from the origin, the set of barriers at the origin, the simplices of the $Q$-system having a vertex at the origin, the $V$-cell $\operatorname{VC}(D)$ at the origin, the Voronoi cell $\Omega(D)$ at the origin, and so forth. In fact, the indexing sets in the definition of the decomposition star were chosen specifically to encode these structures.

\subsection{Colored Spaces}

In Section 3 we introduced a function $\sigma$ that will be formally defined in Definition 7.8. The details of the definition of $\sigma$ are not needed for the discussion that follows. The function $\sigma$ on the space DS of decomposition stars is continuous. This section gives an alternate description of the sense in which this function is continuous.

We begin with an example that illustrates the basic issues. Suppose that we have a discontinuous piecewise linear function on the unit interval $[-1,1]$, as in Fig. 6.1. It is continuous, except at $x=0$.

We break the interval in two at $x=0$, forming two compact intervals $[-1,0]$ and $[0,1]$. We have continuous functions $f_{-}:[-1,0] \rightarrow \mathbb{R}$ and $f_{+}:[0,1]$, such that

$$
f(x)= \begin{cases}f_{-}(x), & x \in[-1,0], \\ f_{+}(x), & \text { otherwise. }\end{cases}
$$

We have replaced the discontinuous function by a pair of continuous functions on smaller intervals, at the expense of duplicating the point of discontinuity $x=0$. We view this pair of functions as a single function $F$ on the compact topological space with two

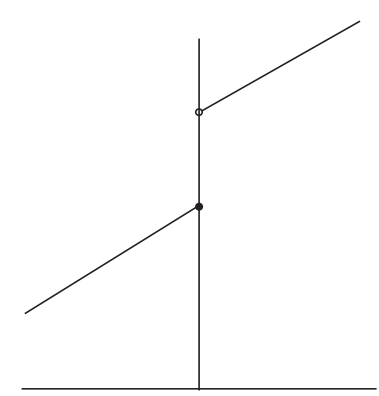

Fig. 6.1. A piecewise linear function. 
components

$$
[-1,0] \times\{-\} \text { and }[0,1] \times\{+\}
$$

where $F(x, a)=f_{a}(x)$ and $a \in\{-,+\}$.

This is the approach that we follow in general with the Kepler conjecture. The function $\sigma$ is defined by a series of case statements, and the function does not extend continuously across the boundary of the cases. However, in the degenerate cases that land precisely between two or more cases, we form multiple copies of the decomposition star for each case, and place each case into a separate compact domain on which the function $\sigma$ is continuous.

This can be formalized as a colored space. A colored space is a topological space $X$ together with an equivalence relation on $X$ with the property that no point $x$ is equivalent to any other point in the same connected component as $x$. We refer to the connected components as colors, and call the points of $X$ colored points. We call the set of equivalence classes of $X$ the underlying uncolored space of $X$. Two colored points are equal as uncolored points if they are equivalent under the equivalence relation.

In our example, there are two colors "- " and "+." The equivalence class of $(x, a)$ is the set of pairs $(x, b)$ with the same first coordinate. Thus, if $x \neq 0$, the equivalence class contains one element $(x, \operatorname{sign}(x))$, and in the boundary case $x=0$ there are two equivalent elements $(0,-)$ and $(0,+)$.

In our treatment of decomposition stars, there are various cases: whether an edge has length less than or greater than $2 t_{0}$, less than or greater than $\sqrt{8}$, whether a face has circumradius less than or greater than $\sqrt{2}$, and so forth. By duplicating the degenerate cases (say an edge of exact length $2 t_{0}$ ), creating a separate connected component for each case, and expressing the optimization problem on a colored space, we obtain a continuous function $\sigma$ on a compact domain $X$.

The colorings have in general been suppressed in places from the notation. To obtain consistent results, a statement about $x \in\left[2,2 t_{0}\right]$ should be interpreted as having an implicit condition saying that $x$ has the coloring induced from the coloring on the component containing $\left[2,2 t_{0}\right]$. A later statement about $y \in\left[2 t_{0}, \sqrt{8}\right]$ deals with $y$ of a different color, and no relation between $x$ and $y$ of different colors is assumed at the endpoint $2 t_{0}$.

\section{Scoring}

This section is coauthored by Samuel P. Ferguson and Thomas C. Hales.

In earlier sections, we describe each packing of unit balls by its set $\Lambda \subset \mathbb{R}^{3}$ of centers of the packing. We showed that we may assume that our packings are saturated in the sense that there is no room for additional balls to be inserted into the packing without overlap. Lemma 3.3 shows that the Kepler conjecture follows if for each saturated packing $\Lambda$ we can find a function $A: \Lambda \rightarrow \mathbb{R}$ with two properties: the function is fcc-compatible and it is saturated in the sense of Definition 3.2.

The purpose of the first part of this section is to define a function $A: \Lambda \rightarrow \mathbb{R}$ for every saturated packing $\Lambda$ and to show that it is negligible. The formula defining $A$ consists of a term that is a correction between the volume of the Voronoi cell $\Omega(v)$ and that of 
the $V$-cell $\mathrm{VC}(v)$ and a further term coming from simplices of the $Q$-system that have a vertex at $v$.

A major theorem in this volume will be that this negligible function is fcc-compatible. The proof of fcc-compatibility can be expressed as a difficult nonlinear optimization problem over the compact topological space DS that was introduced in Section 6. In fact, we construct a continuous function $A_{0}$ on the space DS such that for each saturated packing $\Lambda$ and each $v \in \Lambda$, the value of the function $A$ at $v$ is a value in the range of the function $A_{0}$ on DS. In this way, we are able to translate the fcc-compatibility of $A$ into an extremal property of the function $A_{0}$ on the space DS.

The proof of fcc-compatibility is more conveniently couched as an optimization problem over a function that is related to the function $A_{0}$ by an affine rescaling. This new function is called the score and is denoted $\sigma$. (The exact relationship between $A_{0}$ and $\sigma$ appears in Definition 7.12.) The function $\sigma$ is a continuous function on the space DS. This function is defined in the final paragraphs of this section.

\subsection{Definitions}

For every saturated packing $\Lambda$, and $v \in \Lambda$, there is a canonically associated decomposition star $D(v, \Lambda)$. The negligible function $A: \Lambda \rightarrow \mathbb{R}$ that we define is a composite

$$
A=A_{0} \circ D(\cdot, \Lambda): \Lambda \rightarrow \mathrm{DS} \rightarrow \mathbb{R}, \quad v \mapsto D(v, \Lambda) \mapsto A_{0}(D(v, \Lambda)),
$$

where $A_{0}: \mathrm{DS} \rightarrow \mathbb{R}$ is as defined by (7.2) and (7.6) below. Each simplex in the $Q$-system with a vertex at $v$ defines by translation to the origin a simplex in the $Q$-system with a vertex at 0 attached to $D(v, \Lambda)$. Let $\mathcal{Q}_{0}(D)$ be this set of translated simplices at the origin. This set is determined by $D$.

Definition 7.1. Let $Q$ be a quarter in $\mathcal{Q}_{0}(D)$. We say that the context of $Q$ is $(p, q)$ if there are $p$ anchors and $p-q$ quarters along the diagonal of $Q$. Write $c(Q, D)$ for the context of $Q \in \mathcal{Q}_{0}(D)$.

Note that $q$ is the number of "gaps" between anchors around the diagonal. For example, the context of a quarter in a quartered octahedron is $(4,0)$. The context of a single quarter is $(2,1)$.

The function $A_{0}$ will be defined to be a continuous function on DS of the form

$$
A_{0}(D)=-\operatorname{vol}(\Omega(D))+\operatorname{vol}(\operatorname{VC}(D))+\sum_{Q \in \mathcal{Q}_{0}(D)} A_{1}(Q, c(Q, D), 0) .
$$

Thus, the function $A_{0}$ measures the difference in volume between the Voronoi cell and the $V$-cell, as well as certain contributions $A_{1}$ from the $Q$-system. The function $A_{1}(Q, c, v)$ depends on $Q$, its context $c$, and a vertex $v$ of $Q$. The function $A_{1}(Q, c, v)$ will not depend on the second argument when $Q$ is a quasi-regular tetrahedron. (The context is not defined for such simplices.)

Definition 7.2. An orthosimplex consists of the convex hull of $\left\{0, v_{1}, v_{1}+v_{2}, v_{1}+\right.$ $\left.v_{2}+v_{3}\right\}$, where $v_{2}$ is a vector orthogonal to $v_{1}$, and $v_{3}$ is orthogonal to both $v_{1}$ and $v_{2}$. We 


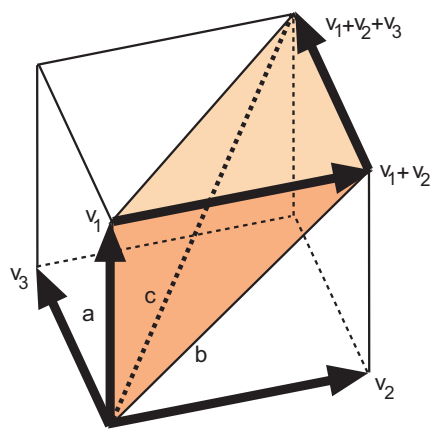

Fig. 7.1. The Rogers simplex is an orthosimplex.

can specify an orthosimplex up to congruence by the parameters $a=\left|v_{1}\right|, b=\left|v_{1}+v_{2}\right|$, and $c=\left|v_{1}+v_{2}+v_{3}\right|$, where $a \leq b \leq c$. This parametrization of the orthosimplex departs from the usual parametrization by the lengths $\left|v_{1}\right|,\left|v_{2}\right|,\left|v_{3}\right|$. For $a \leq b \leq c$, the Rogers simplex $R(a, b, c)$ is an orthosimplex of the form

$$
R(a, b, c)=S\left(a, b, c, \sqrt{c^{2}-b^{2}}, \sqrt{c^{2}-a^{2}}, \sqrt{b^{2}-a^{2}}\right) .
$$

See Fig. 7.1.

Definition 7.3. Let $R$ be a Rogers simplex. We define the quoin of $R$ to be the wedgelike solid (a quoin) situated above $R$. It is defined as the solid bounded by the four planes through the faces of $R$ and a sphere of radius $c$ at the origin. (See Fig. 7.2.) We let quo $(R)$ be the volume of the quoin over $R$. If $R=R(a, b, c)$ is a Rogers simplex, the volume quo $(R)$ is given explicitly as follows:

$$
\begin{aligned}
6 \text { quo }(R)= & (a+2 c)(c-a)^{2} \arctan (e)+a\left(b^{2}-a^{2}\right) e \\
& -4 c^{3} \arctan (e(b-a) /(b+c)),
\end{aligned}
$$

where $e \geq 0$ is given by $e^{2}\left(b^{2}-a^{2}\right)=\left(c^{2}-b^{2}\right)$.

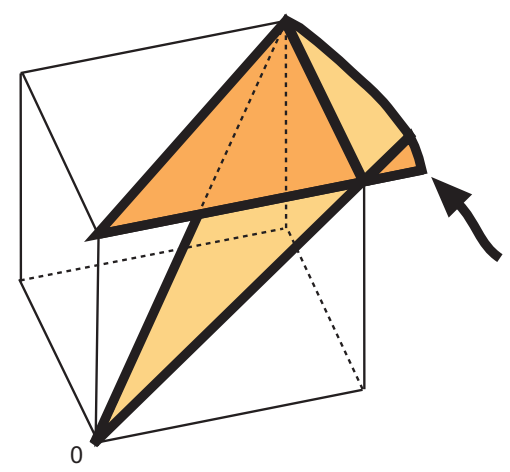

Fig. 7.2. The quoin above a Rogers simplex is the part of the shaded solid outside the illustrated box. It is bounded by the shaded planes, the plane through the front face of the box, and a sphere centered at the origin passing through the opposite corner of the box. 
Let $S$ be a simplex and let $v$ be a vertex of that simplex. Let $\operatorname{VC}(S, v)$ be the subset of $|S|$ consisting of points closer to $v$ than to any other vertex of $S$. By Lemma 5.28, if $S \in \mathcal{Q}_{0}(D)$, then

$$
\operatorname{VC}(S, 0)=\operatorname{VC}(D) \cap|S| .
$$

Under the assumption that $S$ contains its circumcenter and that every one of its faces contains its circumcenter, an explicit formula for the volume $\operatorname{vol}(\operatorname{VC}(S, v))$ has been calculated in Section 8.6.3 of [Ha6]. This volume formula is an algebraic function of the edge lengths of $S$, and may be analytically continued to give a function of $S$ with chosen vertex $v$ :

$$
\operatorname{vol} \operatorname{VC}^{\mathrm{an}}(S, v) .
$$

Lemma 7.4. Let $B(0, t)$ be a ball of radius $t$ centered at the origin. Let $v_{1}$ and $v_{2}$ be vertices. Assume that $\left|v_{1}\right|<2 t$ and $\left|v_{2}\right|<2 t$. Truncate the ball by cutting away the caps

$$
\operatorname{cap}_{i}=\left\{x \in B(0, t):\left|x-v_{i}\right|<|x|\right\} .
$$

Assume that the circumradius of the triangle $\left\{0, v_{1}, v_{2}\right\}$ is less than $t$. Then the intersection of the caps, $\operatorname{cap}_{1} \cap$ cap $_{2}$, is the union of four quoins.

Proof. This is true by inspection. See Fig. 7.3. Slice the intersection cap ${ }_{1} \cap \operatorname{cap}_{2}$ into four pieces by two perpendicular planes: the plane through $\left\{0, v_{1}, v_{2}\right\}$, and the plane perpendicular to the first and passing through 0 and the circumcenter of $\left\{0, v_{1}, v_{2}\right\}$. Each of the four pieces is a quoin.

Definition 7.5. Let $v \in \mathbb{R}^{3}$ and let $X$ be a measurable subset of $\mathbb{R}^{3}$. Let $\operatorname{sol}(X, v)$ be the area of the radial projection of $X \backslash\{0\}$ to the unit sphere centered at the origin. We call this area the solid angle of $X($ at $v)$. When $v=0$, we write the function as $\operatorname{sol}(X)$.

Let $S=\left\{v_{0}, v_{1}, v_{2}, v_{3}\right\}$ be a simplex. Fix $t$ in the range $t_{0} \leq t \leq \sqrt{2}$. Assume that $t$ is at most the circumradius of $S$. Assume that it is at least the circumradius of each of the

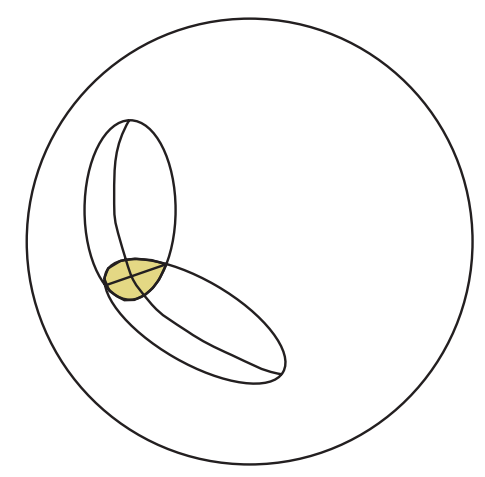

Fig. 7.3. The intersection of two caps on the unit ball can be partitioned into four quoins (shaded). 
faces of $S$. Let $\mathrm{VC}_{t}\left(S, v_{0}\right)$ be the intersection of $\mathrm{VC}\left(S, v_{0}\right)$ with the ball $B\left(v_{0}, t\right)$. Under the assumption that $S$ contains its circumcenter and that every one of its faces contains it circumcenter, an explicit formula for the volume

$$
\operatorname{vol}\left(\mathrm{VC}_{t}\left(S, v_{0}\right)\right)
$$

is calculated by means of Lemma 7.4 through a process of inclusion and exclusion. In detail, start with $|S| \cap B\left(v_{0}, t\right)$. Truncate this solid by caps: cap 1 , cap $_{2}$, and cap counded $_{3}$ by the sphere of radius $t$ centered at $v_{0}$ and the perpendicular bisectors (respectively) of $\left\{v_{0}, v_{1}\right\},\left\{v_{0}, v_{1}\right\}$, and $\left\{v_{0}, v_{2}\right\}$. If we subtract the volume of each cap, cap ${ }_{i}$, then we must add back the volume of the doubly counted intersections of the caps. The intersections of caps are given as quoins (Lemma 7.4). This leads to the following formula. Let $h_{i}=\left|v_{i}\right| / 2$ and $\eta_{i j}=\eta\left(0, v_{i}, v_{j}\right)$, and let $S_{3}$ be the group of permutations of $\{1,2,3\}$ in

$$
\operatorname{vol~} \operatorname{VC}_{t}\left(S, v_{0}\right)=\frac{\operatorname{sol}(S)}{3}-\sum_{i=1}^{3} \frac{\operatorname{dih}\left(S, v_{i}\right)}{2 \pi} \operatorname{vol} \operatorname{cap}_{i}+\sum_{(i, j, k) \in S_{3}} \operatorname{quo}\left(R\left(h_{i}, \eta_{i j}, t\right)\right)
$$

We extend formula (7.4) by setting

$$
\operatorname{quo}(R(a, b, c))=0
$$

if the constraint $a<b<c$ fails to hold. Similarly, set vol cap ${ }_{i}=0$ if $\left|v_{i}\right| \geq 2 t$. With these conventions, formula (7.4) extends to all simplices. We write the extension of $\operatorname{vol} \operatorname{VC}_{t}(S, v)$ as

$$
\operatorname{vol~} \mathrm{VC}_{t}^{+}(S, v) \text {. }
$$

Definition 7.6. Let $^{3}$

$$
\begin{aligned}
\mathrm{s}-\operatorname{vor}(S, v) & =4\left(-\delta_{\mathrm{oct}} \operatorname{vol~VC} \mathrm{VC}^{\mathrm{an}}(S, v)+\operatorname{sol}(S, v) / 3\right), \\
\mathrm{s}-\operatorname{vor}(S, v, t) & =4\left(-\delta_{\mathrm{oct}} \operatorname{vol} \mathrm{VC}_{t}^{+}(S, v)+\operatorname{sol}(S, v) / 3\right),
\end{aligned}
$$

and

$$
\mathrm{s}-\operatorname{vor}_{0}(S, v)=\mathrm{s}-\operatorname{vor}\left(S, v, t_{0}\right) \text {. }
$$

When it is clear from the context that the vertex $v$ is fixed at the origin, we drop $v$ from the notation of these functions. If $S=\left\{v_{1}, v_{2}, v_{3}, v_{4}\right\}$, we define $\Gamma(S)$ as the average

$$
\Gamma(S)=\frac{1}{4} \sum_{i=1}^{4} \mathrm{~s}-\operatorname{vor}\left(S, v_{i}\right)
$$

The average $\Gamma(S)$ is called the compression of $S$.

\footnotetext{
${ }^{3}$ In [Ha1] the volumes in this definition were volumes of Voronoi cells, and hence the notation vor for the function was adopted. We retain vor in the notation, although this direct connection with Voronoi cells has been lost.
} 
Definition 7.7. Let $Q$ be a quarter. Let $\eta^{+}(Q)$ be the maximum of the circumradii of the two faces of $Q$ along the diagonal of $Q$.

Let $Q$ be a simplex in the $Q$-system. We define an involution $v \rightarrow \hat{v}$ on the vertices of $Q$ as follows. If $Q$ is a quarter and $v$ is an endpoint of the diagonal, then let $\hat{v}$ be the opposite endpoint of the diagonal. In all other cases, set $\hat{v}=v$.

We are ready to complete the definition of the function $A: \Lambda \rightarrow \mathbb{R}$. The definition of $A$ was reduced to that of $A_{0}$ in (7.1). The function $A_{0}$ was reduced in turn to that of $A_{1}$ in (7.2). To complete the definition, we define $A_{1}$.

Definition 7.8. Set

$$
A_{1}(S, c, v)=-\operatorname{vol} \operatorname{VC}(S, v)+\frac{\operatorname{sol}(S, v)}{3 \delta_{\text {oct }}}-\frac{\sigma(S, c, v)}{4 \delta_{\text {oct }}},
$$

where $\sigma$ is given as follows:

1. When $S$ is a quasi-regular tetrahedron:

(a) If the circumradius of $S$ is less than 1.41 , set

$$
\sigma(S,-, v)=\Gamma(S) .
$$

(b) If the circumradius of $S$ is at least 1.41, set

$$
\sigma(S,-, v)=\mathrm{s}-\operatorname{vor}(S, v) .
$$

2. When $S$ is a strict quarter:

(a) If $\eta^{+}(S)<\sqrt{2}$ :

(i) If the context $c$ is $(2,1)$ or $(4,0)$, set

$$
\sigma(S, c, v)=\Gamma(S) .
$$

(ii) If the context of $S$ is anything else, set

$$
\sigma(S, c, v)=\Gamma(S)+\frac{\mathrm{s}-\operatorname{vor}_{0}(S, v)-\mathrm{s}-\operatorname{vor}_{0}(S, \hat{v})}{2} .
$$

(b) If $\eta^{+}(S) \geq \sqrt{2}$ :

(i) If the context of $S$ is $(2,1)$, set

$$
\sigma(S, c, v)=\mathrm{s}-\operatorname{vor}(S, v) .
$$

(ii) If the context of $S$ is $(4,0)$, set

$$
\sigma(S, c, v)=\frac{\mathrm{s}-\operatorname{vor}(S, v)+\mathrm{s}-\operatorname{vor}(S, \hat{v})}{2} .
$$

(iii) If the context of $S$ is anything else, set

$$
\begin{aligned}
\sigma(S, c, v)= & \frac{\mathrm{s}-\operatorname{vor}(S, v)+\mathrm{s}-\operatorname{vor}(S, \hat{v})}{2} \\
& +\frac{\mathrm{s}-\operatorname{vor}_{0}(S, v)-\mathrm{s}-\operatorname{vor}_{0}(S, \hat{v})}{2} .
\end{aligned}
$$


When the context and vertex $v$ are given, we often write $\sigma(S)$ or $\sigma(S, v)$ for $\sigma(S, c, v)$.

When $\eta^{+}<\sqrt{2}$, we say that the quarter has compression type. Otherwise, we say it has Voronoi type. To say that a quarter has compression type means that $\Gamma(S)$ is one term of the function $\sigma(S, v)$. It does not mean that $\Gamma(S)$ is equal to $\sigma(S, v)$.

The definition of $\sigma$ on quarters can be expressed a second way in terms of a function $\mu$. If $S$ is a quarter, set

$$
\mu(S, v)= \begin{cases}\Gamma(S), & \text { if } \eta^{+}(S)<\sqrt{2}, \\ \mathrm{~s}-\operatorname{vor}(S, v), & \text { otherwise. }\end{cases}
$$

If $S$ is a flat quarter, we have $\sigma(S, c, v)=\mu(S, v)$, for all contexts $c$.

Suppose $S$ is an upright quarter. Definition 7.8 can be expressed as follows:

- Context $(2,1)$ : set $\sigma(S, c, v)=\mu(S, v)$.

- Context $(4,0)$ : set $\sigma(S, c, v)=(\mu(S, v)+\mu(S, \hat{v})) / 2$.

- Other contexts: set $\sigma(S, c, v)=\left(\mu(S, v)+\mu(S, \hat{v})+\mathrm{s}-\operatorname{vor}_{0}(S, v)-\right.$ $\left.\mathrm{s}-\operatorname{vor}_{0}(S, \hat{v})\right) / 2$.

Lemma 7.9. $\quad A_{0}: \mathrm{DS} \rightarrow \mathbb{R}$ is continuous.

Proof. The continuity of $D \mapsto \operatorname{vol} \mathrm{VC}(D)$ is proved in Lemma 6.6. The continuity of $D \mapsto \operatorname{vol} \Omega(D)$ is similarly proved. The terms vol $\operatorname{VC}(S, v)$ and $\operatorname{sol}(S, v)$ are continuous. To complete the proof we check that the function $\sigma(S, c, v)$ is continuous. It is not continuous when viewed as a function of the set of quarters, because of the various cases breaking at circumradius 1.41 and $\eta^{+}(S)=\sqrt{2}$. However, these cutoffs have been inserted into the data defining a decomposition star (in the indexing sets $I_{8}$ and $I_{9}$ ). Thus, the different cases in the definition of $\sigma(S, c, v)$ land in different connected components of the space DS and continuity is obtained.

We conclude this section with a result that will be of use in the next section.

Lemma 7.10. Let $S=\left\{v_{1}, v_{2}, v_{3}, v_{4}\right\}$ be a simplex in the $S$-system, and let $c$ be its context. Then

$$
\sum_{i=1}^{4} A_{1}\left(S, c, v_{i}\right)=0
$$

Proof. By formula (7.6), this is equivalent to

$$
\sum_{i=1}^{4} \sigma\left(S, c, v_{i}\right)=\sum_{i=1}^{4} \mathrm{~s}-\operatorname{vor}\left(S, c, v_{i}\right) .
$$

Equation (7.8) is evident from Definition 7.8 for $\sigma$. In fact, the terms of the form s-vor 0 have opposing signs and cancel when we sum. The other terms are weighted averages of the terms s-vor $\left(S, c, v_{i}\right)$. Equation (7.8) is thus established because a sum is unaffected by taking weighted averages of its terms. 


\subsection{Negligibility}

Let $B(x, r)$ be the closed ball of radius $r \in \mathbb{R}$ centered at $x$. Let $\Lambda(x, r)=\Lambda \cap B(x, r)$.

Recall from Definition 3.2 that a function $A: \Lambda \rightarrow \mathbb{R}$ is said to be negligible if there is a constant $C_{1}$ such that for all $r \geq 1$,

$$
\sum_{v \in \Lambda(x, r)} A(v) \leq C_{1} r^{2}
$$

Recall the function $A: \Lambda \rightarrow \mathbb{R}$ given by (7.1). Explicitly, let

$$
A(v)=A_{0}(D(v, \Lambda))
$$

where $A_{0}$ in turn depends on functions $A_{1}$ and $\sigma$, as determined by (7.2), (7.6), and Definition 7.8.

Theorem 7.11. The function A of (7.1) is negligible.

Proof. First we consider a simplification, where we replace $A$ with $A^{\prime}$ defined by

$$
A^{\prime}(v, \Lambda)=-\operatorname{vol}(\Omega(D(v, \Lambda)))+\operatorname{vol}(\operatorname{VC}(D(v, \Lambda))) .
$$

(That is, at first we ignore the function $A_{1}$.) The Voronoi cells partition $\mathbb{R}^{3}$, as do the $V$-cells. We have $\Omega(v, \Lambda) \subset B(v, 2)$ (by saturation) and $\operatorname{VC}(v, \Lambda) \subset B(v, 2 \sqrt{3}$ ) (by Definition 5.5). Hence the Voronoi cells with $v \in \Lambda(x, r)$ cover $B(x, r-2)$. Moreover, the $V$-cells with $v \in \Lambda(x, r)$ are contained in $B(x, r+2 \sqrt{3})$. Hence

$$
\sum_{v \in \Lambda(x, r)} A^{\prime}(v) \leq-\operatorname{vol} B(x, r-2)+\operatorname{vol} B(x, r+2 \sqrt{3}) \leq C_{1}^{\prime} r^{2}
$$

for some constant $C_{1}^{\prime}$.

If we do not make the simplification, we must include the sum

$$
\sum_{v \in \Lambda(x, r)} \sum_{Q \in \mathcal{Q}_{v}(D(v, \Lambda))} A_{1}(Q, c, v)
$$

Each quarter $Q=\left\{v_{1}, v_{2}, v_{3}, v_{4}\right\}$ in the $Q$-system occurs in four sets $\mathcal{Q}_{v_{i}}\left(D\left(v_{i}, \Lambda\right)\right)$. By Lemma 7.10 the sum cancels, except when some vertex of $Q$ lies inside $\Lambda(x, r)$ and another lies outside. Each such simplex lies inside a shell of width $2 \sqrt{8}$ around the boundary. The contribution of such boundary terms is again bounded by a constant times $r^{2}$. This completes the proof.

\subsection{Fcc-Compatibility}

We have constructed a negligible function $A$. The rest of this volume will prove that this function is fcc-compatible. This section translates fcc-compatibility into a property that will be easier to prove. To begin with, we introduce a rescaled version of the function $A$. 
Definition 7.12. Let $\sigma:$ DS $\rightarrow \mathbb{R}$ be given by

$$
\sigma(D)=-4 \delta_{\text {oct }}\left(\operatorname{vol} \Omega(D)+A_{0}(D)\right)+16 \pi / 3 .
$$

It is called the score of the decomposition star.

Recall from Definition 3.6 the constant $p t \approx 0.05537$. This constant is called a point.

Lemma 7.13. Let $A_{0}, A$, and $\sigma$ be the functions defined by (7.1), (7.2), (7.6), and Definition 7.8. The following are equivalent:

1. The minimum of the function on DS given by

$$
D \mapsto \operatorname{vol} \Omega(D)+A_{0}(D)
$$

is $\sqrt{32}$.

2. The maximum of $\sigma$ on DS is 8 pt.

Moreover, these statements imply

- For every saturated packing $\Lambda$, the function A is fcc-compatible.

(Eventually, we prove fcc-compatibility by proving $\sigma(D) \leq 8 p t$ for all $D \in \mathrm{DS}$.)

Proof. To see the equivalence of the first and second statements, use Definition 7.12, and the identity

$$
8 p t=-4 \delta_{\text {oct }}(\sqrt{32})+16 \pi / 3 .
$$

(Note that this identity is parallel in form to Definition 7.12 for $\sigma$.)

For a given saturated packing $\Lambda$, the function $A$ has the form $A(v)=A_{0}(D(v, \Lambda))$. Also, $\Omega(D(v, \Lambda))$ is a translate of $\Omega(v)$, the Voronoi cell at $v$. In particular, they have the same volume. Thus, $\operatorname{vol} \Omega(v)+A(v)$ lies in the range of the function

$$
\operatorname{vol} \Omega(D)+A_{0}(D)
$$

on DS. The minimum of this function is $\sqrt{32}$ by the first of the equivalent statements. It now follows from the definition of fcc-compatibility, that $A: \Lambda \rightarrow \mathbb{R}$ is indeed fcccompatible.

Theorem 7.14. If the maximum of the function $\sigma$ on DS is $8 p t$, then for every saturated packing $\Lambda$ there exists a negligible fcc-compatible function A.

Proof. This follows immediately from Theorem 7.11 and Lemma 7.13.

\subsection{Scores of Standard Clusters}

The last section introduced a function $\sigma$ called the score. We show that the function $\sigma$ can be expressed as a sum over terms attached to each of the standard regions. 
Definition 7.15. A standard cluster is a pair $(R, D)$ where $D$ is a decomposition star and $R$ is one of its standard regions. A quad cluster is the standard cluster obtained when the standard region is a quadrilateral.

We break $\sigma$ into a sum

$$
\sigma(D)=\sum_{R} \sigma_{R}(D)
$$

indexed by the standard clusters $(R, D)$. Let

$$
\mathrm{VC}_{R}(D)=\mathrm{VC}(D) \cap \operatorname{cone}(R),
$$

whenever $R$ is a measurable subset of the unit sphere. Let

$$
\mathcal{Q}_{0}(R, D)=\left\{Q \in \mathcal{Q}_{0}(D): Q \subset \operatorname{cone}(R)\right\} .
$$

By Lemma 5.26, each $Q$ is entirely contained in the cone over a single standard region.

Definition 7.16. Let $R$ be a measurable subset of the unit sphere. Set

$$
\operatorname{vor}_{R}(D)=4\left(-\delta_{\text {oct }} \operatorname{vol~VC}(D)+\operatorname{sol}(R) / 3\right) .
$$

Let $R$ be a standard region. Set

$$
\sigma_{R}(D)=\operatorname{vor}_{R}(D)-4 \delta_{\text {oct }} \sum_{Q \in \mathcal{\mathcal { Q } _ { 0 }}(R, D)} A_{1}(Q, c(Q, D), 0) .
$$

Lemma 7.17. $\sigma(D)=\sum_{R} \sigma_{R}(D)$, where the sum runs over all standard regions $R$.

Proof.

$$
\begin{aligned}
\sigma(D) & =-4 \delta_{\text {oct }}\left(\operatorname{vol} \Omega(D)+A_{0}(D)\right)+16 \pi / 3 \\
& =-4 \delta_{\text {oct }}\left(\operatorname{vol} \operatorname{VC}(D)+\sum_{Q \in \mathcal{Q}_{0}(D)} A_{1}(Q, c(Q, D), 0)\right)+(4)(4 \pi / 3) \\
& =\sum_{R} 4\left(-\delta_{\text {oct }} \operatorname{vol~VC}_{R}(D)-\delta_{\text {oct }} \sum_{Q \in \mathcal{Q}_{0}(R, D)} A_{1}(Q, c(Q, D), 0)+\operatorname{sol}(R) / 3\right) .
\end{aligned}
$$

Also, we have

$$
\operatorname{vor}(D)=\sum_{R \in \mathcal{R}(D)} \operatorname{vor}_{R}(D)
$$

Lemma 7.18. Let $R^{\prime} \subset R$ be the part of a standard region that does not lie in any cone over any $Q \in Q_{0}(R, D)$. Then

$$
\sigma_{R}(D)=\operatorname{vor}_{R^{\prime}}(D)+\sum_{Q \in \mathcal{Q}_{0}(R, D)} \sigma(Q, c(Q, D), 0) .
$$


Proof. Substitute the definition of $A_{1}$ (7.6) into the definition of $\sigma_{R}(D)$, noting that $\operatorname{VC}(Q, 0)=\operatorname{VC}_{R^{\prime \prime}}(D)$, where $R^{\prime \prime}$ is the intersection of $Q$ with the unit sphere.

Remark 7.19. Lemma 7.18 explains why we have chosen the same symbol $\sigma$ for the functions $\sigma_{R}(D)$ and $\sigma(Q, c, v)$. We can view Lemma 7.18 as asserting a linear relation in the functions $\sigma$ :

$$
\sigma_{R}(D)=\sigma_{R^{\prime}}(D)+\sum \sigma(Q, c, 0) .
$$

The sum runs over $Q \in \mathcal{Q}_{0}$ that lie in the cone over $R$.

\subsection{Scores of Simplices and Cones}

Many of the functions in this paper are defined by terms involving volumes of simple solids. To give estimates on the functions, it is often convenient to partition the solids into smaller pieces and then define corresponding functions on each of the pieces. For this reason, we define some variants of the functions vor and $\sigma$.

Remark 7.20. We now define a few more variants of the function vor. The function s-vor and its truncated version s-vor $(\cdot, t)$ have been defined already. The function $\operatorname{vor}_{R}(D)$ will also be given a truncated version $\operatorname{vor}_{R}(D, t)$, for a real truncation parameter $t \geq 0$. The special case $\operatorname{vor}_{R}\left(D, t_{0}\right)$ will be abbreviated $\operatorname{vor}_{0, R}(D)$. There will be another variant $\mathrm{r}$-vor for Rogers simplices, and another c-vor for general sets. The general form of these functions is

$$
\mathrm{c}-\operatorname{vor}(X)=4\left(-\delta_{\text {oct }} \operatorname{vol}(X)+\operatorname{sol}(X) / 3\right),
$$

for any subset $X \subset \mathbb{R}^{3}$. The differences between the different versions of vor come from the different choices of the set $X$ and the way they are parametrized.

Definition 7.21. Let $R=R(a, b, c)$ be a Rogers simplex. Assume that the vertex terminating the edges of lengths $a, b$, and $c$ is situated at the origin. Let

$$
\mathrm{r}-\operatorname{vor}(R)=4\left(-\delta_{\text {oct }} \operatorname{vol}(R)+\operatorname{sol}(R) / 3\right) .
$$

Definition 7.22. Let $C(h, t)$ denote the compact cone of height $h$ and circular base. Set

$$
\varphi(h, t)=2\left(2-\delta_{\text {oct }} t h(h+t)\right) / 3 .
$$

Then

$$
\mathrm{c}-\operatorname{vor}(C(h, t))=2 \pi(1-h / t) \varphi(h, t) .
$$

Remark 7.23. Below, we introduce variants of the function $\sigma$. We have already encountered $\sigma$ in Definitions 7.8, 7.12, and 7.16. Informally, we call $\sigma$ (and various functions that are closely related to it) the score. Equation (7.11) represents the score of $C(h, t)$. 
The solid angle of $C(h, t)$ is $2 \pi(1-h / t)$, so $\varphi(h, t)$ is the score per unit area. Also, $\varphi(t, t)$ is the score per unit area of a ball of radius $t$. That is, $\varphi(t, t)=4\left(-\delta_{\text {oct }} \mathrm{vol} / \mathrm{sol}+\frac{1}{3}\right)$.

We set

$$
\begin{aligned}
\mathrm{s}-\operatorname{vor}(S, t)= & \operatorname{sol}(S) \varphi(t, t)+\sum_{\substack{i=1 \\
h_{i} \leq t}}^{3} d_{i}\left(1-h_{i} / t\right)\left(\varphi\left(h_{i}, t\right)-\varphi(t, t)\right) \\
& -\sum_{(i, j, k) \in S_{3}} 4 \delta_{\mathrm{oct}} \operatorname{quo}\left(R\left(h_{i}, \eta\left(y_{i}, y_{j}, y_{k+3}\right), t\right)\right) .
\end{aligned}
$$

In the definition we adopt the convention that $\operatorname{quo}(R)=0$, if $R=R(a, b, c)$ does not exist (that is, if the condition $0<a \leq b \leq c$ is violated). In the second sum, $S_{3}$ is the set of permutations on three letters. This definition is compatible with Definition 7.6.

Similarly, we define $\operatorname{vor}_{P}(D, t)$ for arbitrary standard clusters $(P, D)$. (We shift notation from $R$ to $P$ for a standard region to avoid conflict with Rogers simplices $R$ in the following definition.) Extending the notation in an obvious way, we have

$$
\begin{aligned}
\operatorname{vor}_{P}(D, t)= & \operatorname{sol}(P) \varphi(t, t)+\sum_{\left|v_{i}\right| \leq 2 t} d_{i}\left(1-\left|v_{i}\right| /(2 t)\right)\left(\varphi\left(\left|v_{i}\right| / 2, t\right)-\varphi(t, t)\right) \\
& -\sum_{R} 4 \delta_{\text {oct }} \operatorname{quo}(R) .
\end{aligned}
$$

The first sum runs over vertices in $P$ of height at most $2 t$. The second sum runs over Rogers simplices $R\left(\left|v_{i}\right| / 2, \eta(F), t\right)$ in $P$, where $F=\left\{0, v_{1}, v_{2}\right\}$ is a face of circumradius $\eta(F)$ at most $t$, formed by vertices in $P$. The constant $d_{i}$ is the total dihedral angle along $\left\{0, v_{i}\right\}$ of the standard cluster. The truncations $t=t_{0}=1.255$ and $t=\sqrt{2}$ will be of particular importance. Set $A(h)=\left(1-h / t_{0}\right)\left(\varphi\left(h, t_{0}\right)-\varphi\left(t_{0}, t_{0}\right)\right)$.

Remark 7.24. We have introduced both untruncated and truncated versions of functions vor and $\sigma$. The truncated versions are used to give upper bounds on the untruncated versions. For example, in the function $\sigma(D)$, the $V$-cell contributes through its volume, as in Remark 7.20. The volume appears with a negative coefficient $-4 \delta_{\text {oct. }}$. Thus, we obtain an upper bound on $\sigma(D)$ by discarding bits of volume from the $V$-cell. This suggests that we might try to give upper bounds on the score $\sigma(D)$ by truncating the $V$-cell in various ways. This is the reason for the truncated versions of these functions.

\subsection{The Example of a Dodecahedron}

Example 7.25. The following example illustrates why better bounds on the density of packings can be obtained with $\sigma(D)$ than with a naive approach based on the volume of Voronoi cells. By scoring quasi-regular tetrahedra with the compression function $\Gamma(S)$ rather than $\mathrm{s}-\operatorname{vor}(S)$, we will find that the score is lowered below $8 p t$ for configurations with many quasi-regular tetrahedra. To work one example, we assume that the decomposition star consists of twelve vertices located at distance 2 from the origin, at the vertices 
of a regular icosahedron. The score is approximately

$20 \Gamma(S(2,2,2,2.10292,2.10292,2.10292)) \approx 1.8 p t<8 p t$.

If s-vor $(S)$ had been used, the score would violate Theorem 3.7:

$$
20 \mathrm{~s}-\operatorname{vor}(S) \approx 13.5493 p t>8 p t .
$$

(This is tied to the fact that the regular dodecahedron of inradius 1 has a smaller volume than the rhombic dodecahedron of inradius 1.)

\section{References}

[CS2] J. H. Conway and N. J. A. Sloane, Sphere Packings, Lattices and Groups, third edition, Springer-Verlag, New York, 1998

[Fej8] L. Fejes Tóth, Lagerungen in der Ebene auf der Kugel und im Raum, second edition, Springer-Verlag, Berlin, 1972.

[Ha1] T. C. Hales, The sphere packing problem, J. Comput. Appl. Math.. 44 (1992), 41-76.

[Ha2] T. C. Hales, Remarks on the density of sphere packings in three dimensions, Combinatorica 13 (1993), 181-187.

[Ha5] T. C. Hales, A reformulation of the Kepler conjecture, Unpublished manuscript, Nov. 1996.

[Ha6] T. C. Hales, Sphere packings, I, Discrete Comput. Geom. 17 (1997), 1-51.

[Ha7] T. C. Hales, Sphere packings, II, Discrete Comput. Geom. 18 (1997), 135-149.

[Ha12] Thomas C. Hales, Cannonballs and honeycombs, Notices Amer. Math. Soc. 47(4) (2000), 440-449.

[Ha13] T. C. Hales, Sphere Packings in 3 Dimensions, Arbeitstagung, 2001.

[Ha14] T. C. Hales, Some algorithms arising in the proof of the Kepler conjecture, in Discrete and Computational Geometry, Algorithms and Combinatorics, vol. 25, Springer-Verlag, Berlin, 2003, pp. 489-507.

[Ha16] T. C. Hales, Computer Resources for the Kepler Conjecture, http://annals.math.princeton.edu/ keplerconjecture/. (The source code, inequalities, and other computer data relating to the solution are also found at http://xxx.lanl.gov/abs/math/9811078v1.)

[Hi] D. Hilbert, Mathematische Probleme, Archiv Math. Phys. 1 (1901), 44-63, also in Mathematical Developments Arising from Hilbert Problems, Proceedings of Symposia in Pure Mathematics, vol. 28, 1976, pp. 1-34.

[Ro1] C. A. Rogers, The packing of equal spheres, Proc. London Math. Soc. (3) 8 (1958), 609-620.

Received November 11, 1998, and in revised form September 12, 2003, and July 25, 2005.

Online publication February 27, 2006. 